Fiber-Optic Displacement Sensors on the Hunters Trophy UGT Impulse Gauge Experiments

Ray E. L. Green

Andrew I. Poutiatine 


\section{DISCLAIMER}

This report was prepared as an account of work sponsored by an agency of the United States Government. Neither the United States Government nor any agency thereof, nor any of their employees, make any warranty, express or implied, or assumes any legal liability or responsibility for the accuracy, completeness, or usefulness of any information, apparatus, product, or process disclosed, or represents that its use would not infringe privately owned rights. Reference herein to any specific commercial product, process, or service by trade name, trademark, manufacturer, or otherwise does not necessarily constitute or imply its endorsement, recommendation, or favoring by the United States Government or any agency thereof. The views and opinions of authors expressed herein do not necessarily state or reflect those of the United States Government or any agency thereof. 


\section{DISCLAIMER}

Portions of this document may be illegible in electronic image products. Images are produced from the best available original document. 


\title{
Fiber-Optic Displacement Sensors on the Hunters Trophy UGT Impulse Gauge Experiments
}

by

\author{
Ray E. L. Green and Andrew I. Poutiatine
}

\begin{abstract}
As part of a program to develop gauges for measurement of various mechanical properties in hostile environments, we fielded purely optical displacement sensors at the ends of long fiber-optic cables as supplements to the regular displacement sensors of four impulse gauges fielded as part of a materials study on the Hunters Trophy underground effects test at the Nevada Test Site. These fiber-optic sensor systems and their performance on the Hunters Trophy test are described in this report.
\end{abstract}

\section{Introduction}

Fiber-optic displacement sensors were inserted as add-on experiments in four of the numerous "pressure cell" impulse gauges [1] fielded by Los Alamos National Laboratory (LANL) in the Hunters Trophy underground test (UGT) conducted by the Defense Nuclear Agency (DNA) at the Nevada Test Site (NTS). These were intended to take concepts $[2,3]$ successfully demonstrated in the Ledoux UGT event $[4,5]$ and incorporate them into economical designs more suited for widespread general application. Most of the instrumentation fielded to operate the sensors was in fact carried over from the Ledoux event [6,7] and is described in a later section. The Hunters Trophy experiments were intended to test the concepts in a much more hostile environment than was encountered in the Ledoux usage. The ultimate goal of this developmental effort is a suite of designs for relatively compact optical displacement sensors suitable for fielding at the end of long runs of optical fiber in areas with severe electrical noise plus other hostile environmental conditions such as high radiation fields. Since the intent of this development effort is easy general application of the designs, we chose to attempt our development work without additional shielding of the equipment beyond that provided by judicious selection of cable routing.

The conditions for which we are designing are assumed to be severe enough that we may not rely on fiber transmission characteristics remaining constant. We also assume that ambient light levels in the region of the sensors can vary dramatically. For displacements on the order of several millimeters such as were of interest for the Ledoux and Hunters Trophy impulse gauges, these assumptions led us to designs based on moving mechanical grids which strongly modulated the throughput levels in a highly collimated light beam supplied and sensed through optical fibers originating in a remote location. By looking for the modulation, independent of the overall light level, one has in principle a digital readout which should function even in the presence of considerable fiber darkening. For the Ledoux sensors where it was possible to calibrate in situ the return light levels as a gauge was exercised, the digital information was augmented with this analog vernier information to refine considerably the level of detail available in the displacement histories measured. For the Hunters Trophy sensors being discussed here we had neither the ability to calibrate in situ nor the luxury of the high post-event fiber transmission seen in Ledoux. High radiation levels forced us to search for digital modulation in very small signals from severely darkened fibers, thus providing an extreme test of this concept. 
Since several systems run by different organizations are involved with the material presented in this report, Table 1 has been included as a guide to the nomenclature associated with the sensors being discussed. For this report, we generally use the electronics support group channel label to identify the sensor or measurement under consideration. The lower entries in the table contain some other experimental details of possible use in future correlation of this report with information from other diverse sources.

Table 1. Guide to the nomenclature used by the various organizations and groups involved with the Hunters Trophy event for the systems associated with the fiber-optic displacement sensor experiments. Some of the other experimental details are also tabulated in the lower portion of the table.

\begin{tabular}{|c|c|c|c|c|c|}
\hline System Description & $\begin{array}{l}\text { Two-fiber } \\
\text { transmission } \\
\text { system \#1 }\end{array}$ & $\begin{array}{l}\text { Two-fiber } \\
\text { transmission } \\
\text { system \#2 }\end{array}$ & $\begin{array}{l}\text { Two-fiber } \\
\text { background } \\
\text { loop }\end{array}$ & $\begin{array}{l}\text { Single-fiber } \\
\text { reflective } \\
\text { system \#1 }\end{array}$ & $\begin{array}{l}\text { Single-fiber } \\
\text { reflective } \\
\text { system \#2 }\end{array}$ \\
\hline $\begin{array}{l}\text { Electronics support group } \\
\text { channel label }\end{array}$ & 45 & 46 & 47 & 48 & 57 \\
\hline $\begin{array}{l}\text { Designation for pressure } \\
\text { cell gauge in which sensor } \\
\text { head was installed }\end{array}$ & P01 & P12 & none & P02 & P08 \\
\hline $\begin{array}{l}\text { First DNA digitizer } \\
\text { unit/channel designation }\end{array}$ & $1508 / 20$ & $1508 / 21$ & $1508 / 22$ & $1508 / 23$ & $1510 / 23$ \\
\hline $\begin{array}{l}\text { Backup DNA digitizer } \\
\text { unit/channel designation }\end{array}$ & $1510 / 20$ & $1510 / 21$ & $1510 / 22$ & none & none \\
\hline $\begin{array}{l}\text { DNA FO-9 cable \#963110, } \\
\text { fiber number }\end{array}$ & $\begin{array}{l}1 \text { in } \\
2 \text { out }\end{array}$ & $\begin{array}{l}5 \text { in } \\
6 \text { out }\end{array}$ & $\begin{array}{l}7 \text { in } \\
8 \text { out }\end{array}$ & 3 & 4 \\
\hline $\begin{array}{l}\text { Vacuum feedthrough, } \\
\text { Polymicro radiation hard } \\
\text { fiber number }\end{array}$ & $\begin{array}{l}1 \text { in } \\
2 \text { out }\end{array}$ & $\begin{array}{l}5 \text { in } \\
7 \text { out }\end{array}$ & $\begin{array}{l}8 \text { in } \\
9 \text { out }\end{array}$ & 3 & 4 \\
\hline Transmitter system & "LD1" & "LD2" & "LD3" & OPTECH & OPTECH \\
\hline Receiver system & $\begin{array}{l}\text { Math Assoc. } \\
\text { serial } 67176\end{array}$ & $\begin{array}{l}\text { Math Assoc. } \\
\text { serial } 67177\end{array}$ & $\begin{array}{l}\text { Math Assoc. } \\
\text { serial } 67199\end{array}$ & $\begin{array}{l}\text { DMM-1A } \\
\text { serial } 103\end{array}$ & $\begin{array}{l}\text { DMM-1A } \\
\text { serial } 101\end{array}$ \\
\hline $\begin{array}{l}\text { DC average pretest signal } \\
\text { level (volts), light sources } \\
\text { on }\end{array}$ & 6.02 & 5.14 & 5.78 & 7.17 & 5.02 \\
\hline $\begin{array}{l}\text { DC average pretest signal } \\
\text { level (volts), light sources } \\
\text { off }\end{array}$ & 0.41 & 1.40 & 0.36 & $n / a$ & $\mathrm{n} / \mathrm{a}$ \\
\hline
\end{tabular}




\section{Sensor Head Designs}

For the Ledoux UGT event, the position sensors that served as forerunners to the present designs used multiple-bit Gray code grids since absolute position sensors were desired for that event and there was adequate space available for the extra bulk involved. For the more general purpose gauges envisioned as the product of the current effort, the goal is a single-bit relative position gauge (in future, possibly read in quadrature for direction information) of relatively compact design, especially in dimensions other than the axis of motion. For the Hunters Trophy UGT event, we did not have a calibrated analog signal level to use as a vernier position measurement so we desired reasonably fine digital resolution. We thus chose a grid size that was just large enough to prevent the interrogation beam from ever sensing two slots at once. This gave the most finely resolved transmitted signal which still had full depth modulation by sacrificing a large, but inconsequential, fraction of the initial interrogation beam. In Ledoux, where the analog vernier measurement was easily available and where we had concerns with tighter light budgets, a larger size was chosen for the finest grid (the least significant bit of the Gray code) to maximize the light throughput while retaining a continuously varying modulation of the transmitted light by the moving grid.

Figures 1 and 2 show the design concepts for the two styles of fiber-optic displacement sensors fielded in Hunters Trophy. The transmission type shown in Fig. 1 used two independent fibers that terminated in identical sets of optical components, namely a graded index microlens (GRIN lens) followed by a miniature prism mirrored to turn the light beam at right angles. All the systems discussed in this report used $100 / 140 \mu \mathrm{m}$ multimode fibers. At the approximately $830 \mathrm{~nm}$ operating wavelength of the optical systems, the graded index lenses used had a pitch of 0.25 so that light in the numerical aperture of a fiber was focused into a nearly parallel beam of approximately $1 \mathrm{~mm}$ diameter (or the inverse process). We used NSG America, Inc. model FCC-3C10G- 0.83 commercial units pigtailed with fiber and sealed in a protective housing by the manufacturer. The locating diameters of the lens housings for these units were $3 \mathrm{~mm}$. The Meadowlark Optics 1/8 inch series prisms used to turn the light beams oriented the fiber cables along the axis of motion and thus dramatically reduced the spatial extent of the gauges in the other directions. The $1.2 \mathrm{~mm}$ by $6.4 \mathrm{~mm}$ cross section moving tongues that attached to the objects of interest slid freely in a rectangular opening of just slightly

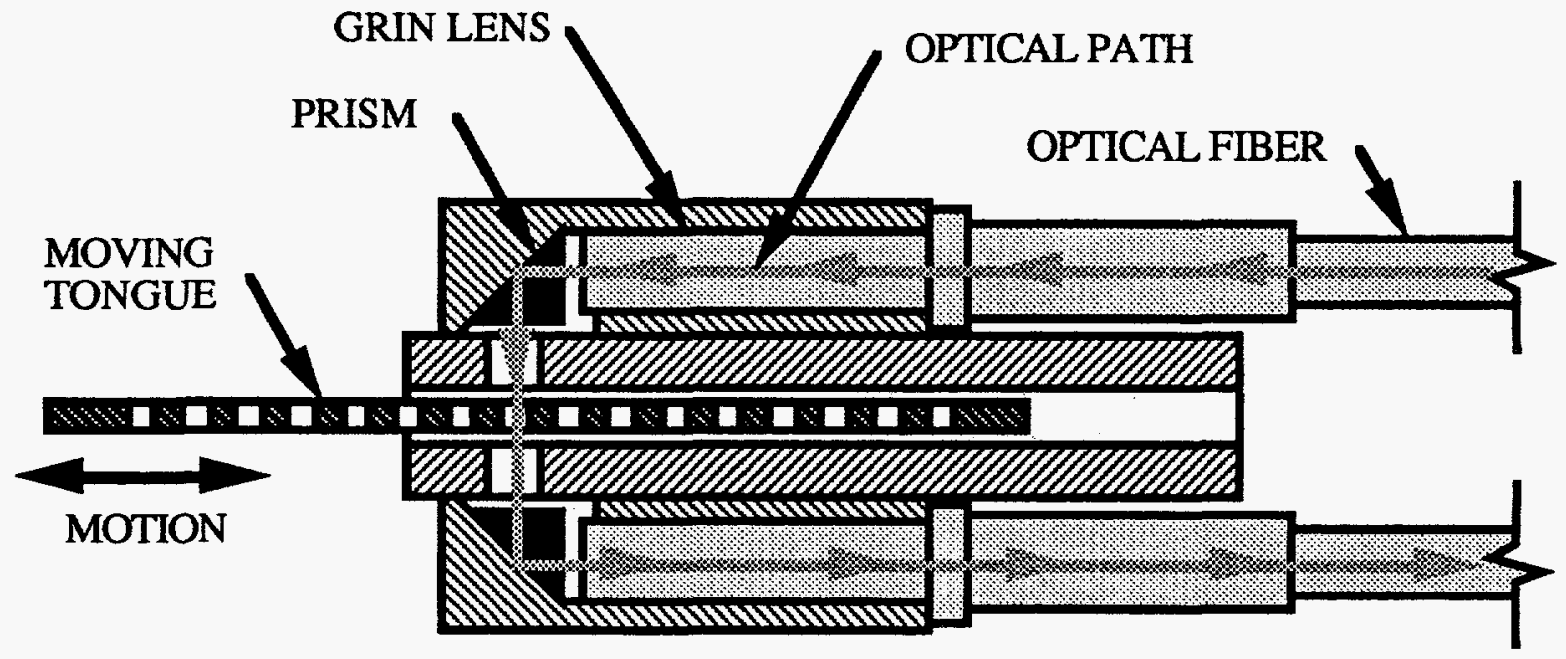

Figure 1. Sketch of section through a transmission gauge sensing head. The input and output sides are identical except for the direction of the light. 
larger dimensions running through the displacement gauge body. The grid pattern to modulate the light consisted of $0.5 \mathrm{~mm}$ holes drilled on $1 \mathrm{~mm}$ centers through the narrow direction of the tongue down most of the length of the center line. As a relative displacement gauge, this design allows for measurements over any length governed only by the length of the tongue. The tongues we used for the Hunters Trophy event had a throw length of approximately $25 \mathrm{~mm}$. The normal displacement sensors in the impulse gauges to which they were attached only had valid operational ranges of approximately $10 \mathrm{~mm}$, but the moving cup assemblies to which they and the optical sensors were attached could move further.

The reflection type sensors of Fig. 2 used identical mechanical modulating grids and had optical component trains on one side of the sensor bodies identical to those of the transmission style sensors. However, the receiving optical system on the other side of the gauge bodies was replaced by a specular reflector such that light emerging from the source fiber was reflected back into it in the absence of any intervening grid. The signals thus were sent back up the fibers which had served as the light sources, to be sorted out by optoelectronic instrumentation described later. This technique reduces the number of fibers required to operate a sensor, but greatly increases background light relative to the signal due to spurious reflections unrelated to modulation grid motion. An older design concept [8] for a very compact reflection style sensor with the graded index lens on the actual axis of a moving stylus containing part of the reflective optics had originally been proposed for some of the Hunters Trophy tests but was abandoned due to its extreme sensitivity to alignment seen during bench tests on prototype gauges.

Even with precision machining of the parts, adequate optical alignment for these systems happened only fortuitously when the parts were bolted together hard. We aligned the optics by placing Belleville spring washers (generally two in reversed orientations) between the main sensor body and the optical component block(s) being attached to it. The Belleville springs were placed on the 2-56 machine screws used and were stiff enough to hold the components securely but had sufficient operating range to allow us to align the optics by maximizing light throughput while adjusting the relative tightness of the machine screws.

The four optical displacement sensors used in Hunters Trophy were each mounted in one of the pressure cell impulse gauges [1] fielded as part of this event. The moving cup in these gauges is normally sensed by a stretch-wire system assembled around the outside of the cup. These were left intact, but the block of crushable honeycomb material

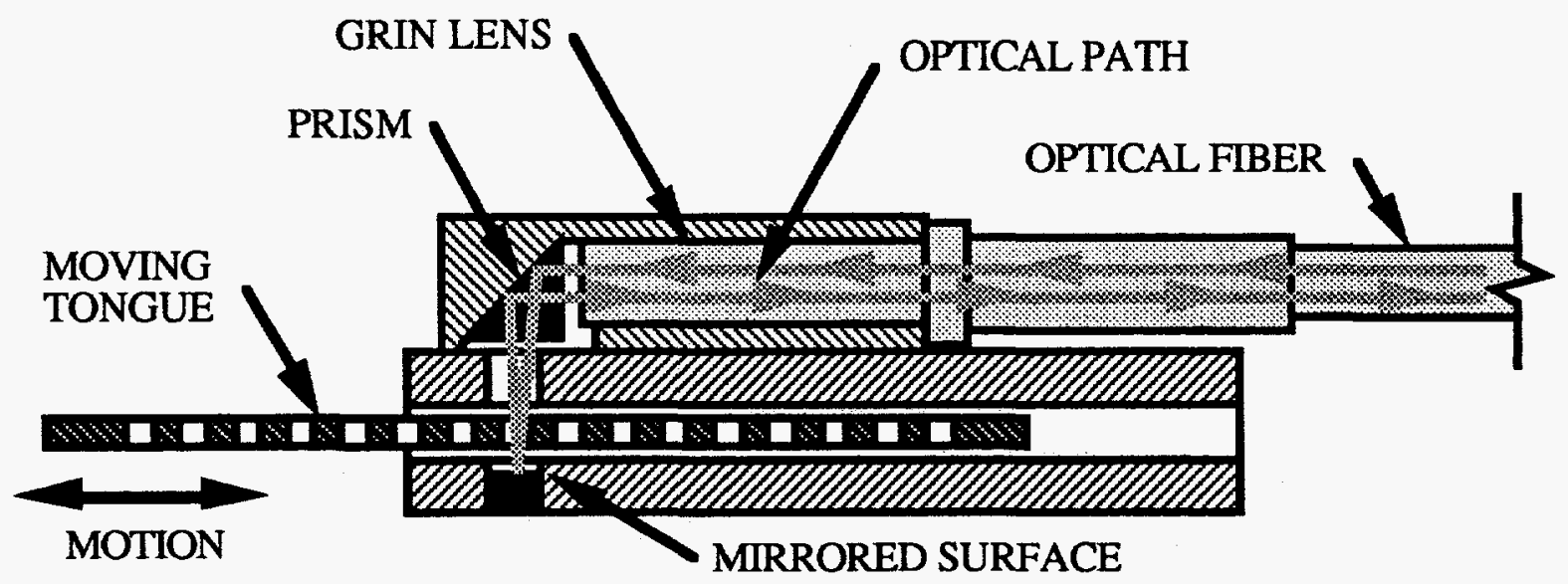

Figure 2. Sketch of section through a reflection gauge sensing head. The output side of the transmission style gauge is replaced with a highly reflective surface and the input optical leg also serves as the receiver for the output optical signal. 
normally mounted in back of the cup beyond the motion range sensed by the stretch-wire system was removed for these four units and replaced by the optical systems and associated mounting hardware. The movable tongue of each optical gauge was aligned with the axis of its pressure cell sample cup and attached such that the initial position corresponded to a maximum in optical throughput thus indicating alignment over a hole in the grid pattern. Once installed in the Hunters Trophy test chamber, there was no access to the sensor heads and no way of exercising the moving parts before the event.

\section{Instrumentation}

Figure 3 is a schematic diagram of the instrumentation used for the Hunters Trophy fiber-optic displacement sensor tests. For the two transmission system sensors, the light sources were Spectra Diode model SDL-2421-H2 pigtailed laser diodes powered by Liconix model LDD100 drivers. The return light, to be modulated by the movement of the grids in the gauges, was sensed in Math Associates model RA-1000/15047E optical receivers. (Since their ultimate frequency response was not required, series resistors of $475 \Omega$ were added to the outputs of the optical receivers in order to short circuit protect them.) As already mentioned, grids in the gauges had been designed to optimize the digital resolution of the system without significant reduction in the depth of modulation but not to optimize optical transmission since the laser diode systems provided significant reserve capacity (we used about $3 \mathrm{~mW}$ out of the $100 \mathrm{~mW}$ of available optical power, barely above the "knee" in power versus diode current that indicates the transition to lasing action). A third background system routed identically to the gauge systems but merely coupled together near the gauge unit connections was also employed. Since it did not sacrifice part of the light as was done in the gauge design, it was necessary for us to insert an attenuator in the line in order to reduce the light level to a value the receivers could tolerate.

The other two gauge units used mirrors on the far side of the modulation grids to reflect light back into the single interrogation fiber that served both as the source and the receiver. These systems were sensed with Optical Technologies model DMM-1A optical transceivers. Since only a small portion of the reflected light seen by the receivers in these units originated from the modulated source of interest (most arose from surfaces in connectors, Rayleigh backscattering in the fibers, and unmodulated reflections in the gauges themselves), these transceivers were designed to subtract the signal from the constant sources of reflected light from that of the total return light and then to amplify this small signal as the output to the data acquisition system. Systems such as those used in Ledoux, where the displacement gauges had built-in motion calibration devices, can be set up with relative ease. For the Hunters Trophy systems, where there was no possibility of exercising the motions of the real gauges after installation and prior to the event, setup using substituted prototypes inserted in place of the actual gauges proved much trickier and less accurate. Given the final results for fiber-optic gauge system performance in Hunters Trophy, these transceiver systems should probably be dropped from consideration for use in such extreme environments, especially if no in situ calibration is possible.

Should a future analysis of some of the data presented here require a more detailed consideration of the optical cabling paths involved, it is worth noting that the FO-9 cable emerged through concrete at ground level near the right rib (right side wall when looking at the source) near test chamber number 1 in the line-of-sight drift. It was routed along the wall up to and across the ceiling until it was above the feedthrough flange (port \#161) allocated to these tests. The FO-9 cable then dropped straight to the flange where an optical enclosure box bolted to the flange itself contained the connections to the radiation hard Polymicro fiber jumpers. Excess length in the FO-9 cable was taken up in several coils on the tunnel wall. The Polymicro fiber jumpers ran through an epoxy plug that served as the vacuum seal and then behind bulkhead 


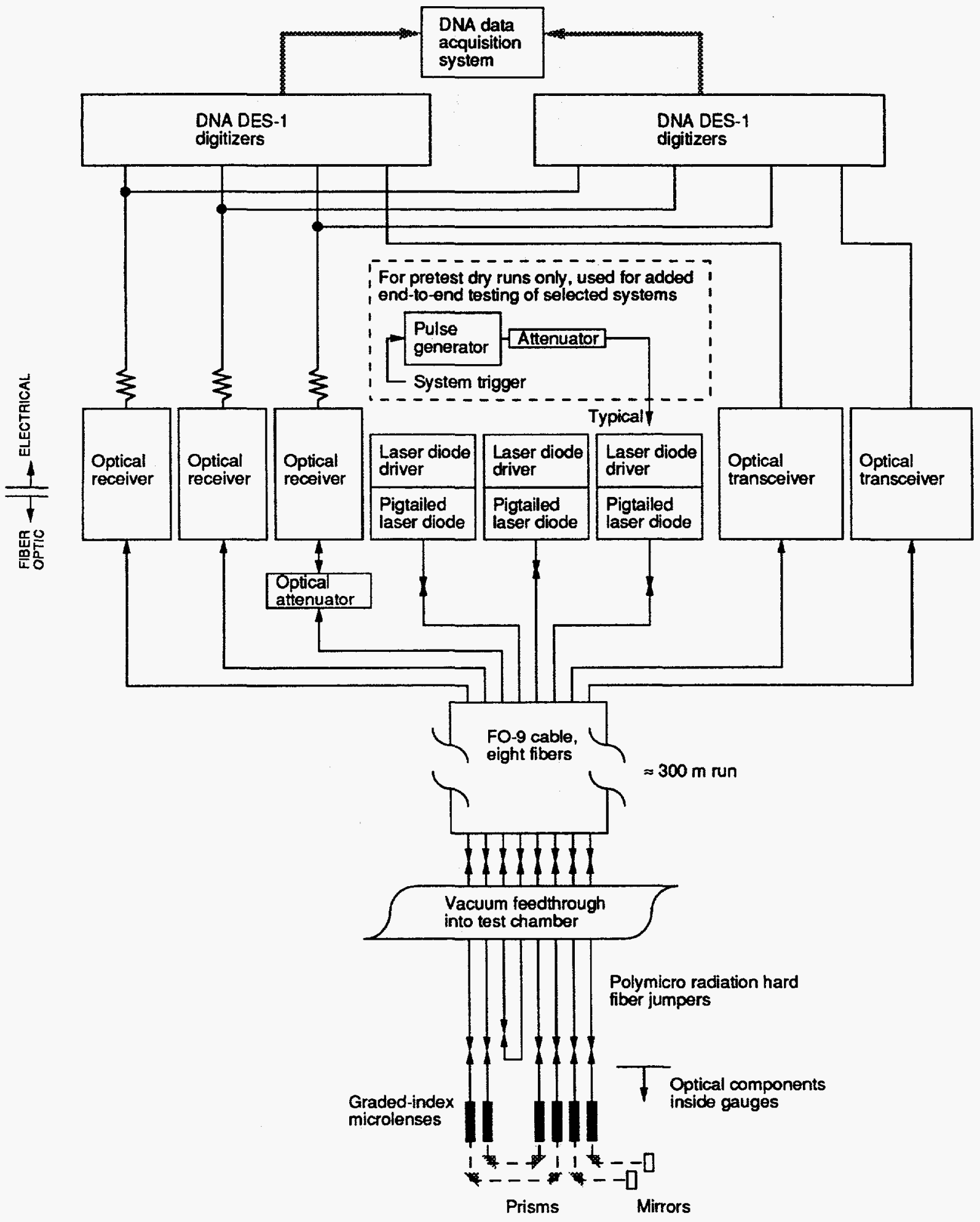

Figure 3. Instrumentation schematic for the Hunters Trophy test of fiber-optic displacement gauges. 
number 12 at scientific station 848 to the backs of the impulse gauge units involved. A small fraction of excess length was coiled up at the flange. The short (about $0.5 \mathrm{~m}$ ) leads from the sensor heads to the Polymicro jumpers were standard graded index fibers as installed by the GRIN lens manufacturer.

Competition among experimenters for the available digitizers led to the choice of DNA DES-1 units operating in the 0-10 volt mode for these experiments. The ability of these units to use different sampling rates during different recording segments was used to successfully overcome their limited memory length. However, their 8-bit resolution may have significantly limited the amount of information ultimately obtained from these tests. (The 12-bit digitizers used for the Ledoux event were a much better match to the capabilities inherent in the rest of the instrumentation.) Setup and running of the digitizers and their interface with the DNA recording station for the Hunters Trophy event were the responsibility of the Command, Control, and Communications Group of Los Alamos National Laboratory [9] with support from EG\&G Energy Measurements, Inc. of Las Vegas [10]. All digitizers and recording systems for the fiber sensor experiments performed as requested for the event.

In order to avoid confusion in interpreting small digitizer signals, the output voltages of the Math Associates optical receivers for no light input were adjusted to provide signals significantly above the digitizer threshold levels. Similarly, the output voltages with the laser diodes on were adjusted to be comfortably within the digitizer range. For light levels between these values, the receivers had been verified to be nearly linear during preparations for the Ledoux event. The gain of the DMM-1A optical transceivers was set by disconnecting the fiber-optic cables from the fielded displacement sensors and inserting a functioning surrogate in place of them. After reconnecting the fielded sensors, the offsets were adjusted to give outputs somewhat above the mid-range

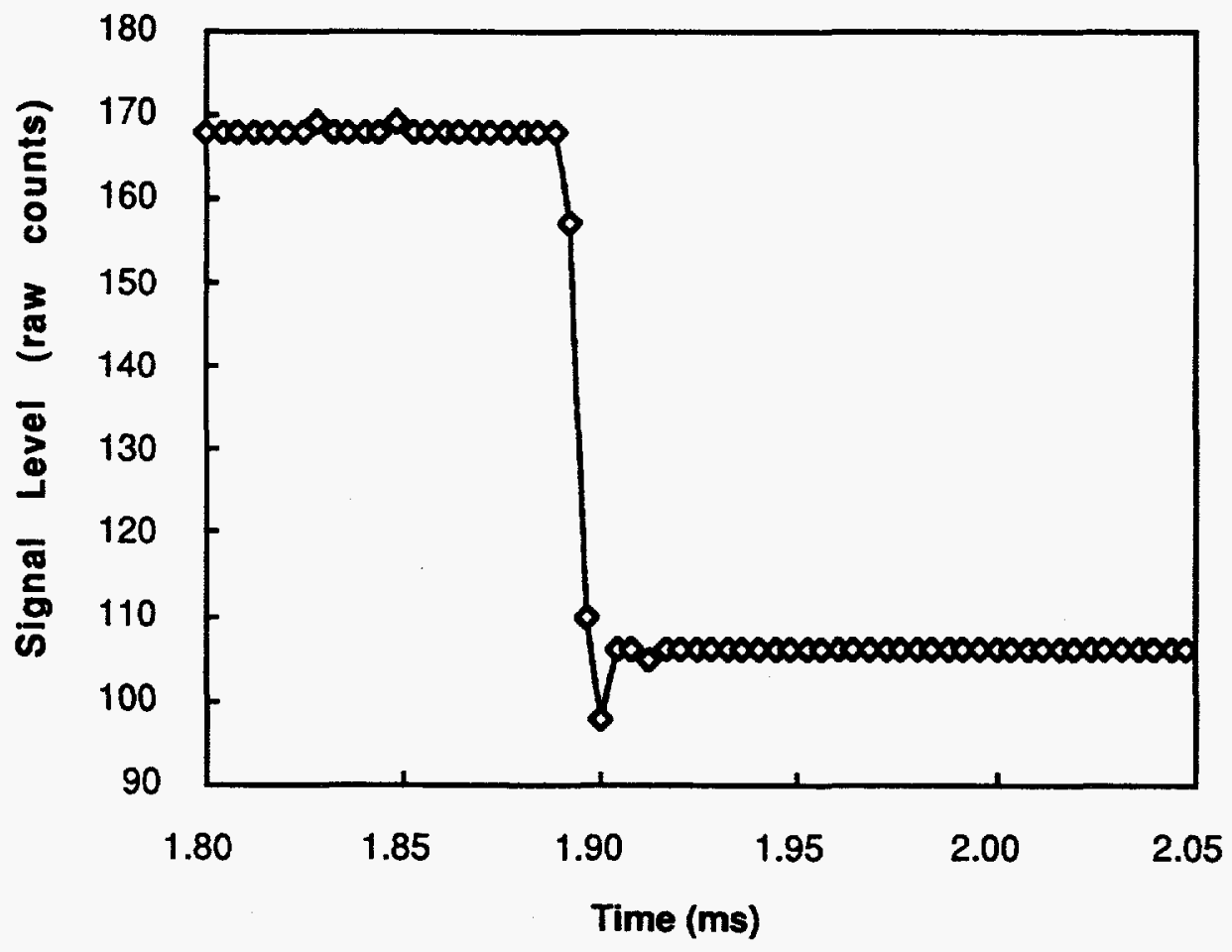

Figure 4. Details of dry run data from the channel 47 background fiber loop for a lightlowering step function injected at the laser diode driver after the master system trigger. 


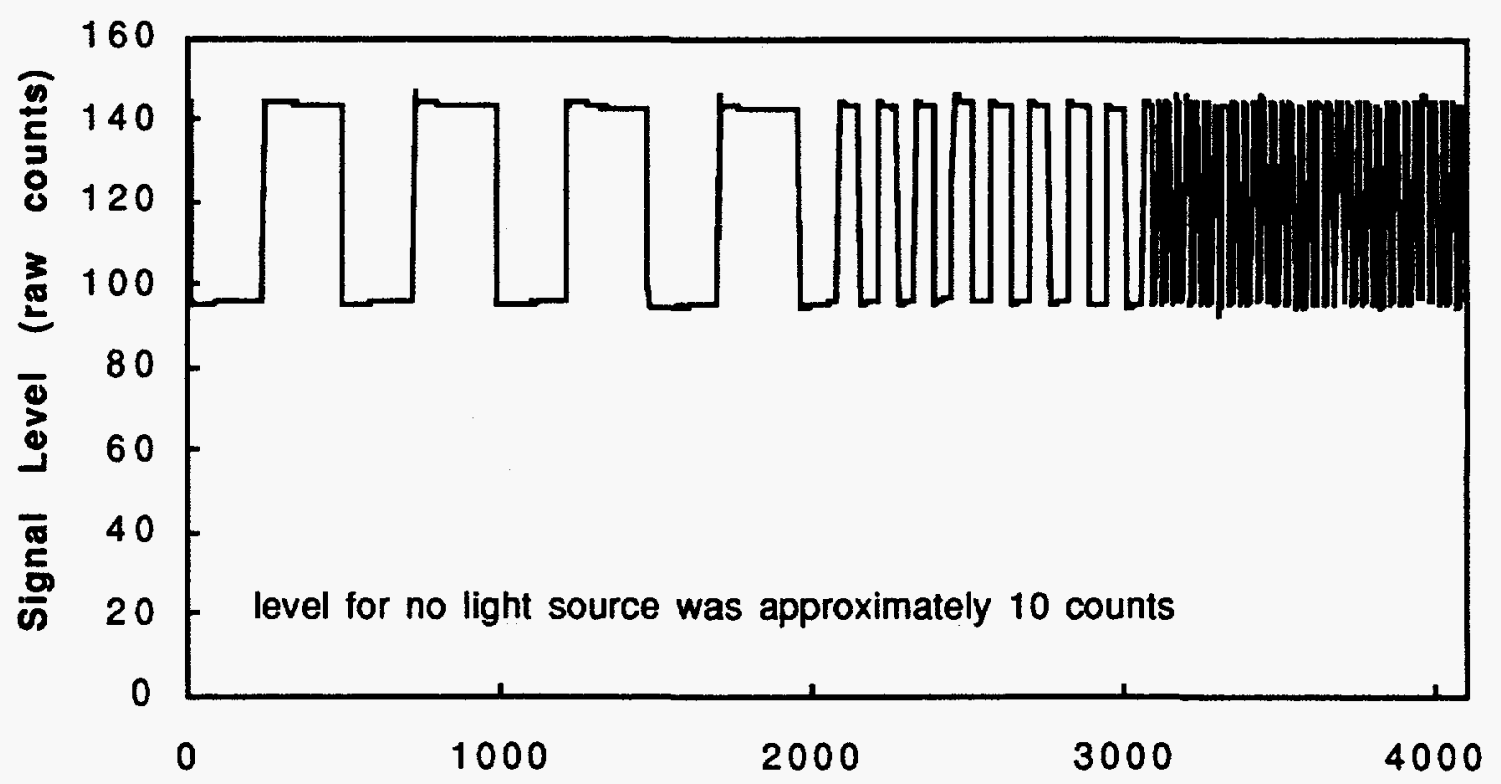

Time Bin Number

Figure 5. Raw data from a dry run for the channel 45 transmission system. A square wave with a period of approximately $2 \mathrm{~ms}$ was injected at the laser diode driver.

of the transceiver output range since we had originally installed the tongues to be close to their maximally reflective positions. The gains and offsets for these systems were large and even an added sharp bend in one of the fibers involved could change its optical characteristics enough to move the signal out of range. Included in Table 1 are the average DC levels provided to the DES-1 digitizers shortly before the test. For the transmission systems, levels with the light sources turned off are also given.

The signal injection system used during some of the dry run tests performed before the event is also indicated in Fig. 3. The output of the Tektronix PG 507 pulse generator used was attenuated $26 \mathrm{~dB}$ to match its range to an operating input range for the drivers that would not result in damage to the laser diodes. Upper and lower output levels from the pulse generator were chosen to give laser diode outputs corresponding to the nominal value chosen for the tests and a reduced value that would still maintain stable operating laser diode characteristics. Signal injection was performed on only one system per dry run, but the chosen system was varied from run to run until operation of all transmission systems had been verified on at least one dry run. To test the timing of our systems relative to the overall UGT system, we used the UGT master trigger from a fan-out unit as the input for the external trigger of our PG 507 pulse generator and introduced a known delay before the pulse output. Figure 4 shows the region of the signal drop in one of the redundant digitizers for such a test. We verified operation of the digitizer at different rates in different quarters of the data record by setting the pulse generator to give free-running nearly square wave modulation between upper and lower levels with a period of approximately $2 \mathrm{~ms}$. Figure 5 shows one such test; data have been parsed to fit display capabilities of the graphics program used.

The time response at the drop in Fig. 4 gives a measure of the combined response of the whole system used, i.e., the pulse generator itself, the laser diode and its driving electronics, and the optical receivers used. The time per point in this portion of the data record is $4 \mu \mathrm{s}$ and signal levels are nearly quiescent in four time steps or $16 \mu \mathrm{s}$ for an 
amplitude step about one-third the total range. Time response of the single-fiber system transceiver units was not tested before the event. From manufacturer specifications, it was known to vary with the gain level but to remain in the tens of kilohertz regime for our application on the Hunters Trophy event, perfectly adequate for the anticipated mechanical responses.

Unlike some of the other systems on Hunters Trophy, the calibration record taken for these systems as part of the main event sequence simply recorded the quiescent initial signal levels in the sensor systems at a sampling frequency of approximately $4.916 \mathrm{kHz}$ for 2285 points just prior to the event. A representative portion showing all four channels of data from one of the digitizer units is given in Fig. 6. The transmission sensor data of channels 45 and 46 both have significant noise in a $60 \mathrm{~Hz}$ repetitive pattern. The noise in the background fiber loop system data of channel 47 appears to be lower than is significant given the 8-bit resolution of the digitizer. The channel 48 data from one of the high-gain reflective sensor systems appears to have a little over \pm 1 bit of random noise. Data from the other digitizer associated with these experiments is similar as would be expected for the three redundantly recorded systems and as happens to be the case for the other similar reflective sensor system.

\section{Data Reduction}

For final data reduction, we started with data sets extracted [11] from the DNA NTS Area 12 general data acquisition system for experimenters and processed them to

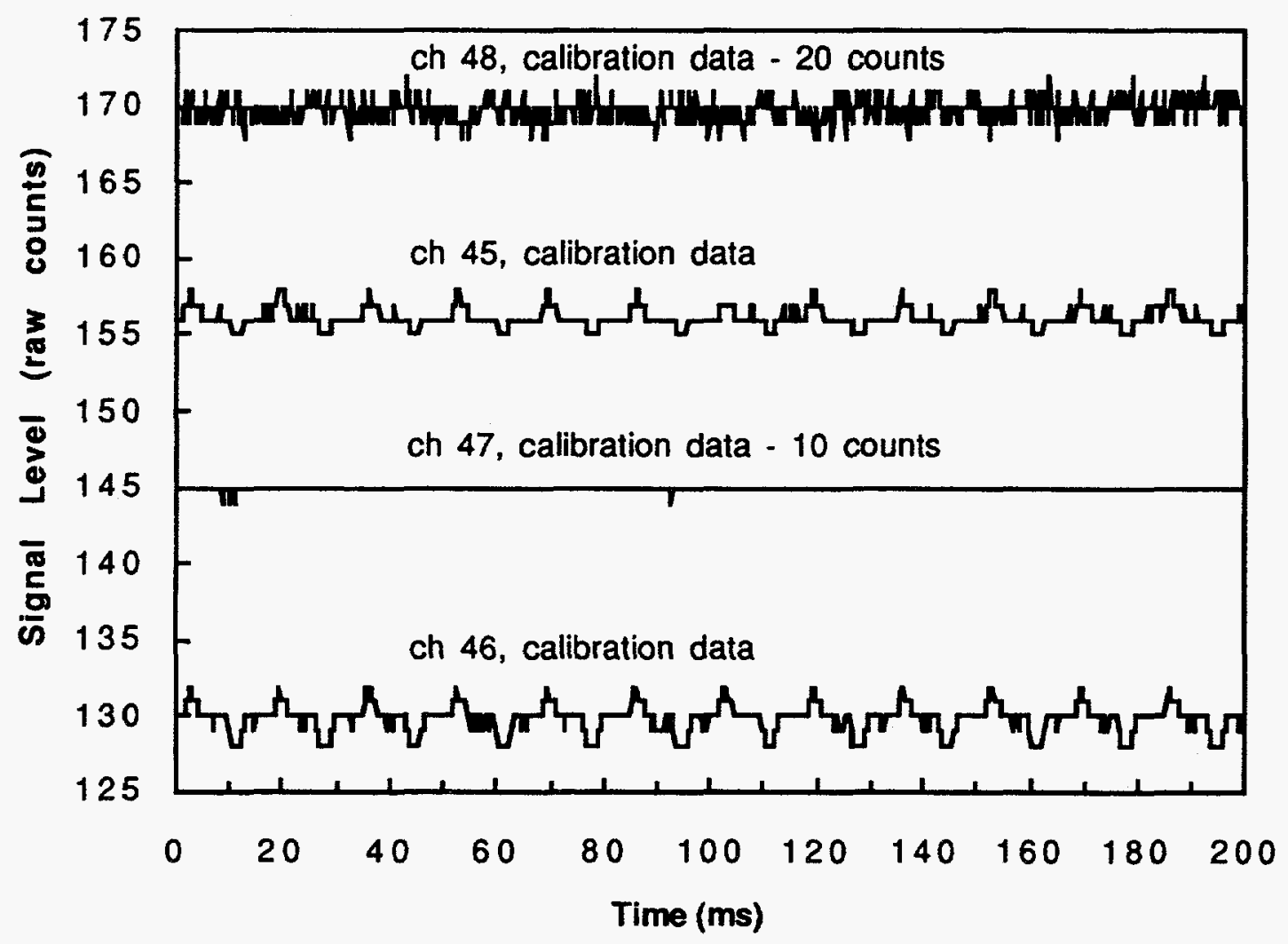

Figure 6. First $200 \mathrm{~ms}$ of similar data from the entire calibration for digitizer 1508 , taken just prior to the event. Some traces are offset by the amounts shown. 
provide time versus raw digitizer bit readings. The actual voltage levels are irrelevant to the analysis and we did not put the calibration for them into the data records since they merely made it more difficult to identify the effects from the coarse binning of the DES-1A digitizers used. The electronics for the reflective sensor systems had limited time resolution and the data were not recorded redundantly. Given the final results, to be discussed later, no further processing was done on the reflective sensor data and it was used in raw form. For the transmission sensor systems and the fiber loop system, more extensive processing was undertaken, driven mainly by the possibility of extracting information from the ragged data of one of the sensor systems. The remainder of this section discusses this processing.

Figure 7 shows parsed (to fit the display capabilities), unprocessed event data from one of the two redundant digitizers on the transmission sensor of most interest. For this figure and all subsequent figures covering the same time span, the short region containing data before the event is hard to discern on this scale and we have arbitrarily extended the average pre-event level to $-1 \mathrm{~ms}$ to enhance graphical clarity. Both this data and that from the other transmission sensor indicate zero light throughput at late times but with $60 \mathrm{~Hz}$ noise signals superimposed on the average signals. The pre-event calibration data of Fig. 6 indicate similar magnitude $60 \mathrm{~Hz}$ noise superimposed on the high-level average signals for these channels. All indications are that this noise originates in the output drivers of the two optical receiver units involved. The data from the four digitizers involved were all corrected for the periodic noise by fitting a five parameter function of appropriate periodicity to the data between $40 \mathrm{~ms}$ and the end of the trace, subtracting this function from all of the data, and rounding to integer values (to maintain the same "graininess" inherent in the raw data). Details of this procedure are contained in Appendix A. The optical receiver unit for the fiber loop system (channel 47) did not have

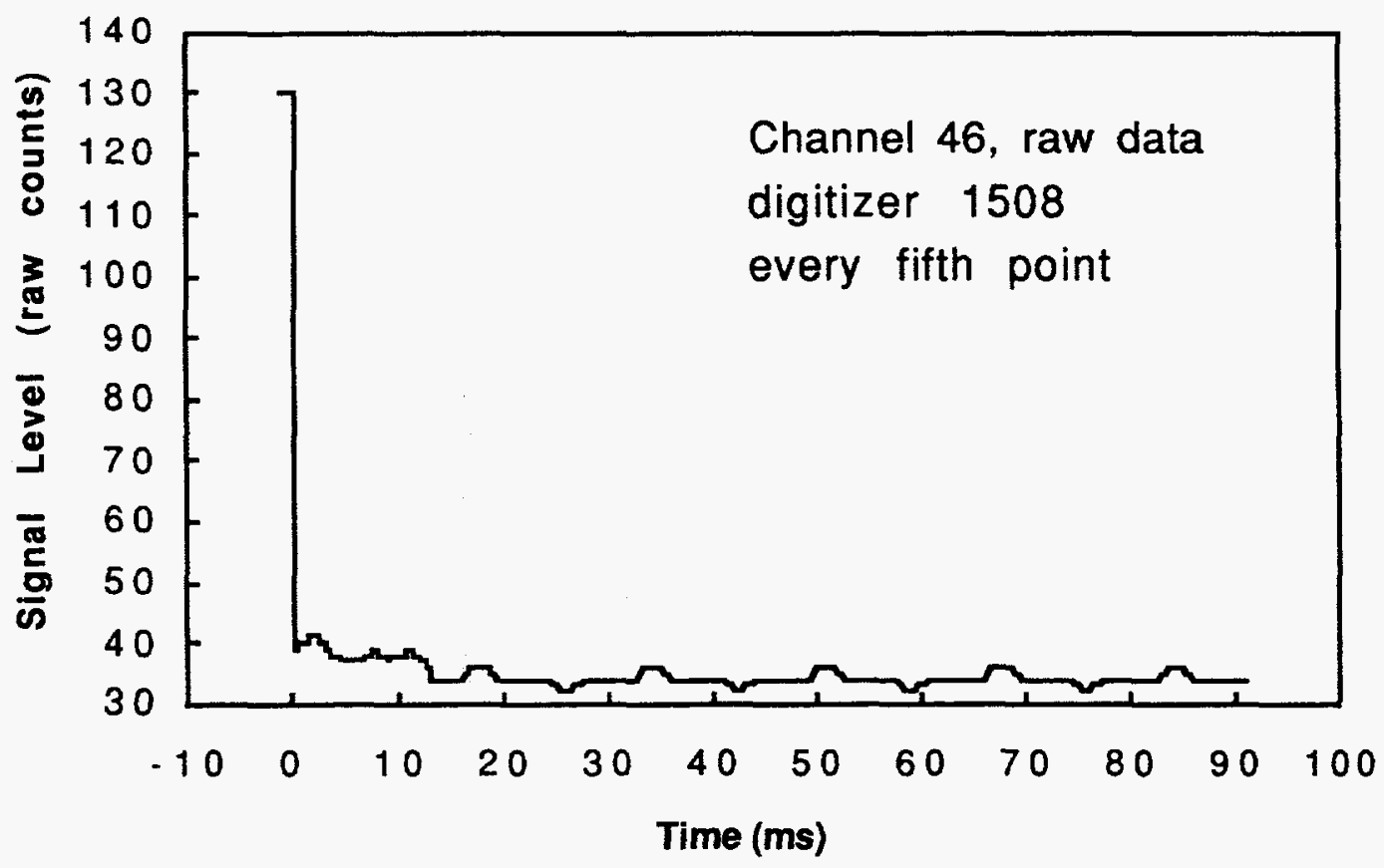

Figure 7. Raw data from one of the two redundant digitizers on the channel 46 transmission gauge system. Note signal between 0 and $13 \mathrm{~ms}$ over and above the $60 \mathrm{~Hz}$ noise pattern. 
the same noise problem and no corrections for $60 \mathrm{~Hz}$ noise were made to the data for either of its digitizers.

The data for the transmission style sensors and the background loop are readily interpreted by using light intensity throughput $\mathrm{I}(\mathrm{t})$ calculated from the noise-corrected data $C(t)$ using

$$
I(t)=\left[C(t)-C_{o f f}\right] /\left[C_{i n i t}-C_{o f f}\right]
$$

where $C_{\text {off }}$ is nominally the digitizer reading before the event with the light source off and $C_{i n i t}$ is the digitizer reading before the event (supposedly at or near maximum transmission) with the light source on. In practice, the $\mathrm{C}_{\text {off }}$ values for each of the digitizers for the channel 45 and 46 data were calculated during the noise correction

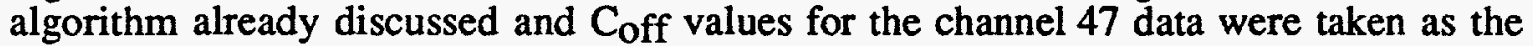
lowest values seen in the data for a given digitizer. The $\mathrm{C}_{\text {off }}$ levels taken from the data were identical within the digitizer resolution to the levels indicated by the DC average values measured when we briefly turned off the light sources just before the diagnostics

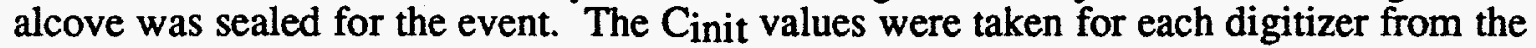
pre-event portion of the noise-corrected data for channels 45 and 46 and from the raw data for channel 47 . Transmission system data presented in the rest of this report use or are derived from these $I(t)$ values. In addition, data from both digitizers for a given system are averaged to enhance detail somewhat, although this is at best a poor substitute for a single digitizer with another bit of resolution. Except for the few points during the very rapid change in level near the beginning of the event, the data from redundant digitizers differ from each other by at most one bit (after the minor corrections for small differences in conversion gain automatically built into the data reduction scheme just described). We also have averaged (rather than parsed as in Fig. 7) the transmission system data subsequently displayed over five raw time steps to further aid in smoothing the data and bringing up details of potential interest. No structures in the data that were not consistent with mere instrumental response as seen in Fig. 4 were obscured by this averaging.

Some details of the data manipulation and a guide to the computer records and file formats as they currently exist are given in Appendix B.

\section{Results for the Background Fiber Loop}

The data in Fig. 8 for transmission through the background fiber loop are probably the most useful information to come from this study, following the recovery of transmission through the fiber link from near zero shortly after the start of the event to $37 \%$ at $91 \mathrm{~ms}$. The two gauges with transmission sensors and this system all used high light levels and low gain receivers and we saw absolutely no indication of fiber flash in any of these systems at either the raw time resolution or when using the five-point averaged data.

The choice of zeros as the lowest readings seen in the digitizer records, while in agreement with the values measured before the test alcove was sealed, is somewhat arbitrary and it seems reasonable to ascribe a one bit uncertainty to it. The lowest readings in both digitizers occurred for only a single time point, one at $12 \mu \mathrm{s}$ after the event began and the other many time steps away at $292 \mu \mathrm{s}$ after the event began. In Fig. 9, we have plotted the effects of a one bit shift in both directions on the data beyond $100 \mu \mathrm{s}$ and have plotted these two limits as $\mathrm{dB}$ loss versus time. According to arguments summarized by Stringer et al. $[12,13]$ and supported by experimental data from pulsed reactor tests discussed by these authors, recovery is expected to have a $\mathbf{t}^{-\mathrm{X}}$ time 


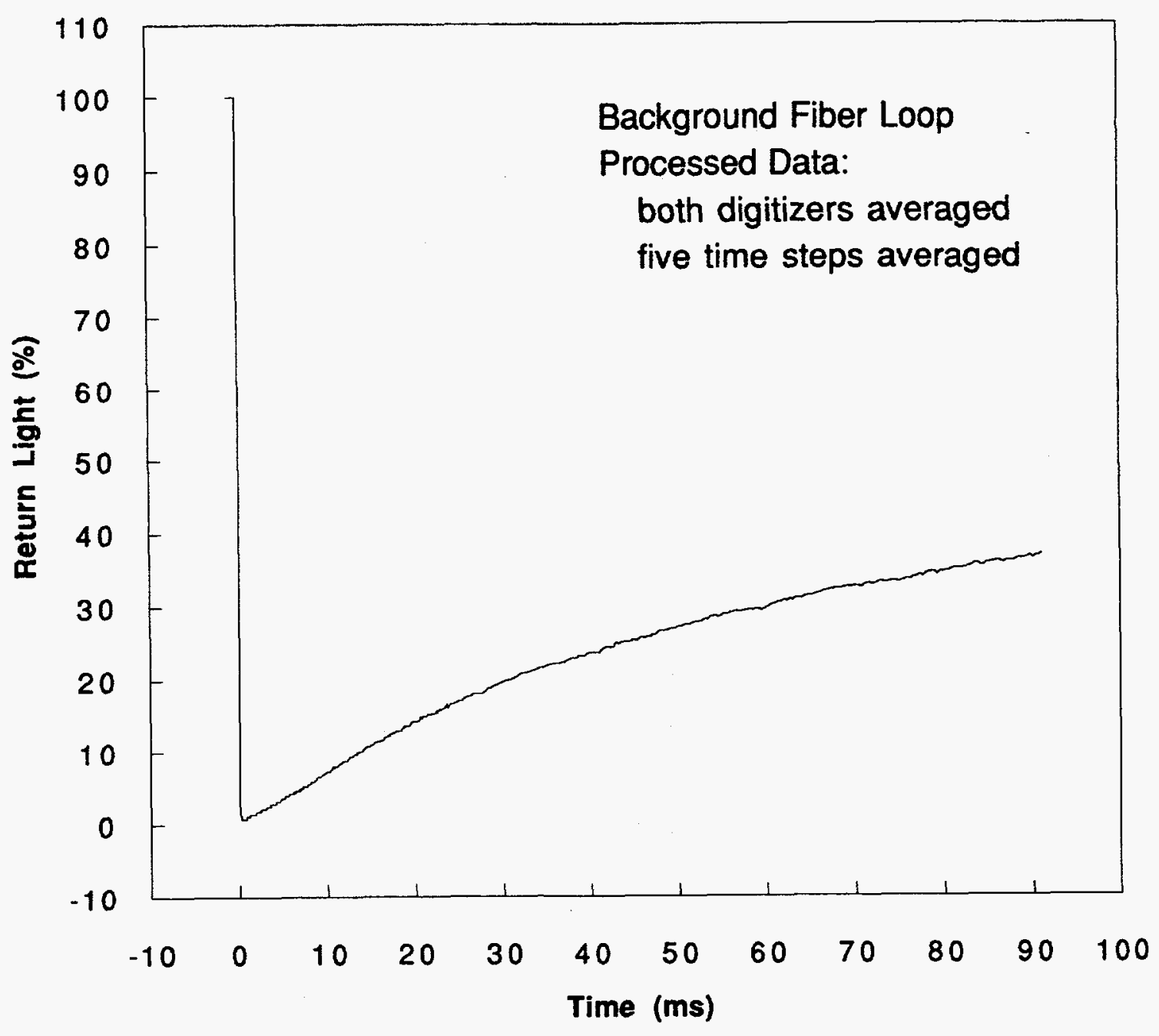

Figure 8. Data for the channel 47 background measurement of transmission through a fiber loop, averaged over redundant digitizers and over five raw time steps. 


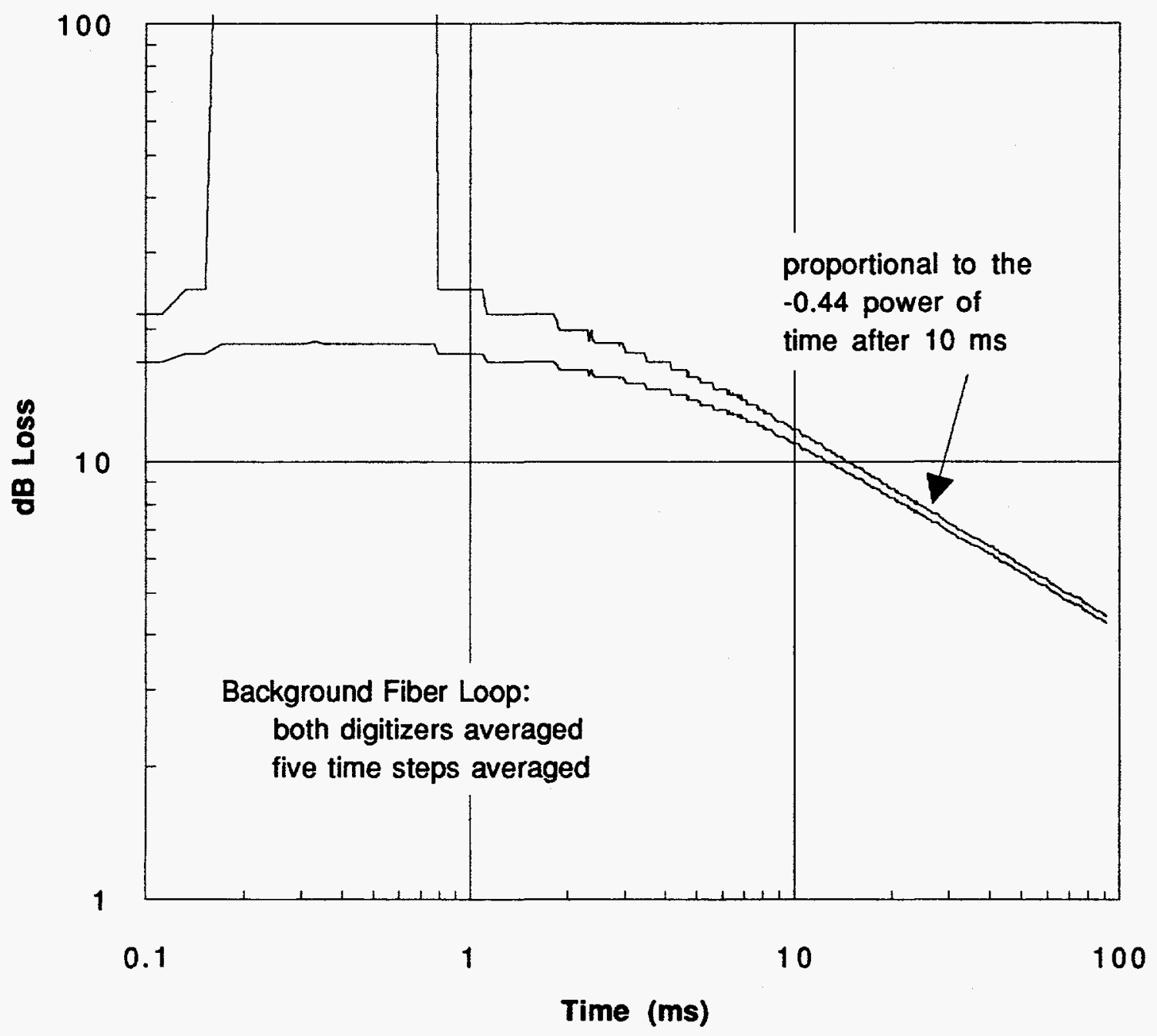

Figure 9. Uncertainty limits for fiber loop transmission loss assuming a \pm 1 bit error in digitizer values for zero light transmission. 
dependence with $0.25<x<0.5$. For the data in Fig. 9 beyond $10 \mathrm{~ms}$, there is a good fit to a $\mathrm{t}^{-0.44}$ dependence.

\section{Results for Reflection Sensor Systems}

Given the extreme darkening of the fibers seen in the background fiber loop system, there cannot be reflection sensor data on a modulated signal originating from mechanical motion of the gauge tongue since the transceiver background level subtraction will have moved the entire signal region completely out of range. However, because of the higher gains involved in these systems, we may see one feature of interest in the early time data displayed for both reflection sensor gauges in Fig. 10. The raw data points are displayed as symbols while the solid lines are fits to the data assuming that the return light seen by the transceivers is constant until fiber flash increases the light level for two points followed by light levels giving no signal (below the background subtraction level), all as modified by a digital exponential smoothing filter to model the frequency response of the electronics. Parameters of the fits for each system are the starting level, the initial point for the increased flash, independent amplitudes in each of the two contiguous flash points, and the filter frequency. The flash amplitudes cannot be interpreted quantitatively because we have no measure of the gains or absolute magnitudes of the return light. In fact, the model should be taken as only suggestive rather than an attempt to accurately account for the details of fiber flash and electronic response if indeed that is even what we are observing (the need to spike two time points which fall slightly later than the onset of fiber darkening in the transmission systems

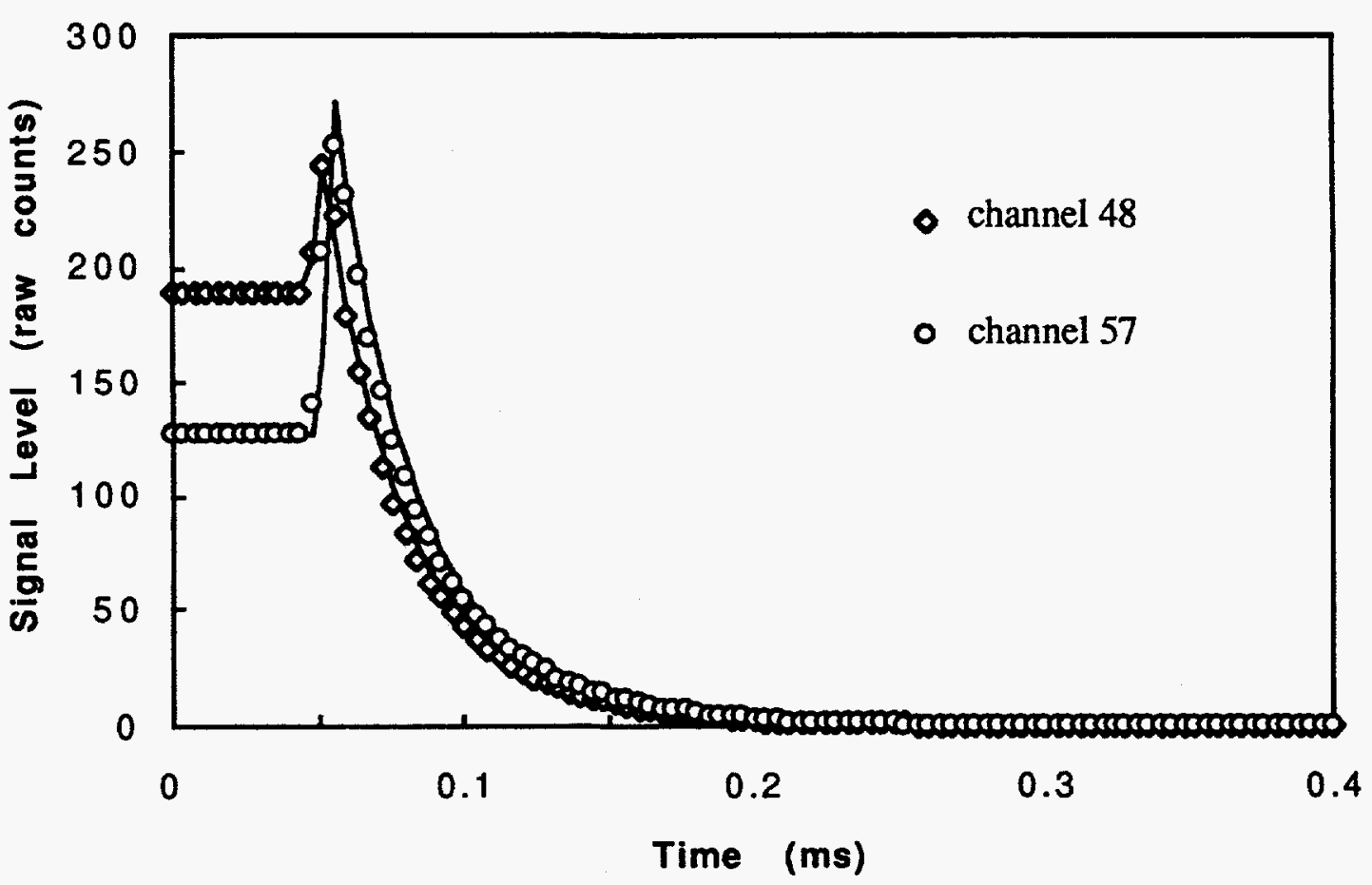

Figure 10. Data from reflective system gauges with fits from a simple model for a short flash in the fibers followed by instantaneous darkening and subsequent processing through $35 \mathrm{kHz}$ electronics. 
suggests we are partially modeling electronic response with our choice of the prefiltered input). The filter frequency used for the fits was $35 \mathrm{kHz}$, falling in the range expected for the transceiver units. The actual value for the frequency response may not be that for ordinary signals in these systems since we are probably seeing the effects of rapid toggling between signals extremely out of range in opposite directions.

\section{Results for Transmission Sensor Systems}

Our original desire was to install the optical displacement gauges in systems where we would have valid input data over the approximately $10 \mathrm{~mm}$ operational range of the host gauges for time windows in the neighborhood of $10 \mathrm{~ms}$, as seen in some of the pressure-cell gauge data from previous UGT events. Unfortunately, the vagaries of making low-priority, non-interfering add-on measurements coupled with the experimental variability associated with the primary measurements precluded such ideal conditions and we ended up testing our fiber-optic systems under even more extreme conditions than contemplated in the introduction. We eventually abandoned our initial attempt to interpret the transmission gauge data without knowledge of the pressure cell results, thus giving up the secondary goal of these experiments to serve as an independent check on the standard pressure-cell sensor performance.

Figure 11 shows our data for the channel 45 system associated with the pressure cell P01. Had there been displacement in the 5 to $10 \mathrm{~ms}$ region still within the active length of the sensor tongue, the background loop data of Figs. 8 and 9 suggest that we should have been able to detect a modulated signal unless the additional darkening of the optical elements inside the gauge bodies was significant. There is clearly no evidence of such a signal and in fact there is no indication of return light at any point after the initial event. Without invoking the P01 pressure cell data, we hypothesized several candidate explanations for the lack of return light. After consulting this data, we decided we were most likely simply out of the sensor range well before fiber transmission was significantly larger than the equivalent of one digitizer bit. The stretch wires in the standard P01 sensor had exceeded their range and broken at approximately $1.1 \mathrm{~ms}$ into this very energetic event [11].

Figure 12 shows our data for the channel 46 system associated with the pressure cell P12. Here we at least see what appears to be return light in the region of interest, expanded in Fig. 13, although it is not easy to fully interpret this signal even after the pressure cell data are consulted. The pressure cell data for this case [11] indicate that the stretch wires slowed the moving cup of the impulse gauge to a stop at about $4.6 \mathrm{~ms}$. At this point, the stretch wires relax and begin periodically shorting out yielding mere noise for their signals and indicating a cup loose in its housing potentially free to move somewhat and perhaps vibrate in response to general motion of the experimental chamber bulkhead. This provides a qualitative explanation for the data of Fig. 13 if we assume that increasing fiber transmission allows us to see the beginnings of modulated signals shortly before the cup stops and then again between 7 and $13 \mathrm{~ms}$ in response to some type of cup vibration or relaxation motion. There are, however, some possible mysteries associated with this interpretation. First, total return light in the first few milliseconds is higher than one would expect given the data on the fiber background loop. The easiest explanation for this would be that the initial position of the tongue in the gauge was not quite centered at maximum transmission (the initial light levels were not set at the top of the digitizer range for just such an eventuality). The second mystery is why the late time signal tails off to zero. It seems extremely fortuitous to assume that the cup just happens to come to a stop at one of the relatively sharp minima in the tongue's transmission grid and it is not clear that the cup and stretch wire assembly have enough freedom with unbroken wires to relax to a region out of range for the tongue in the optical displacement sensor. The third mystery, given the significant depth of modulation by the optical 


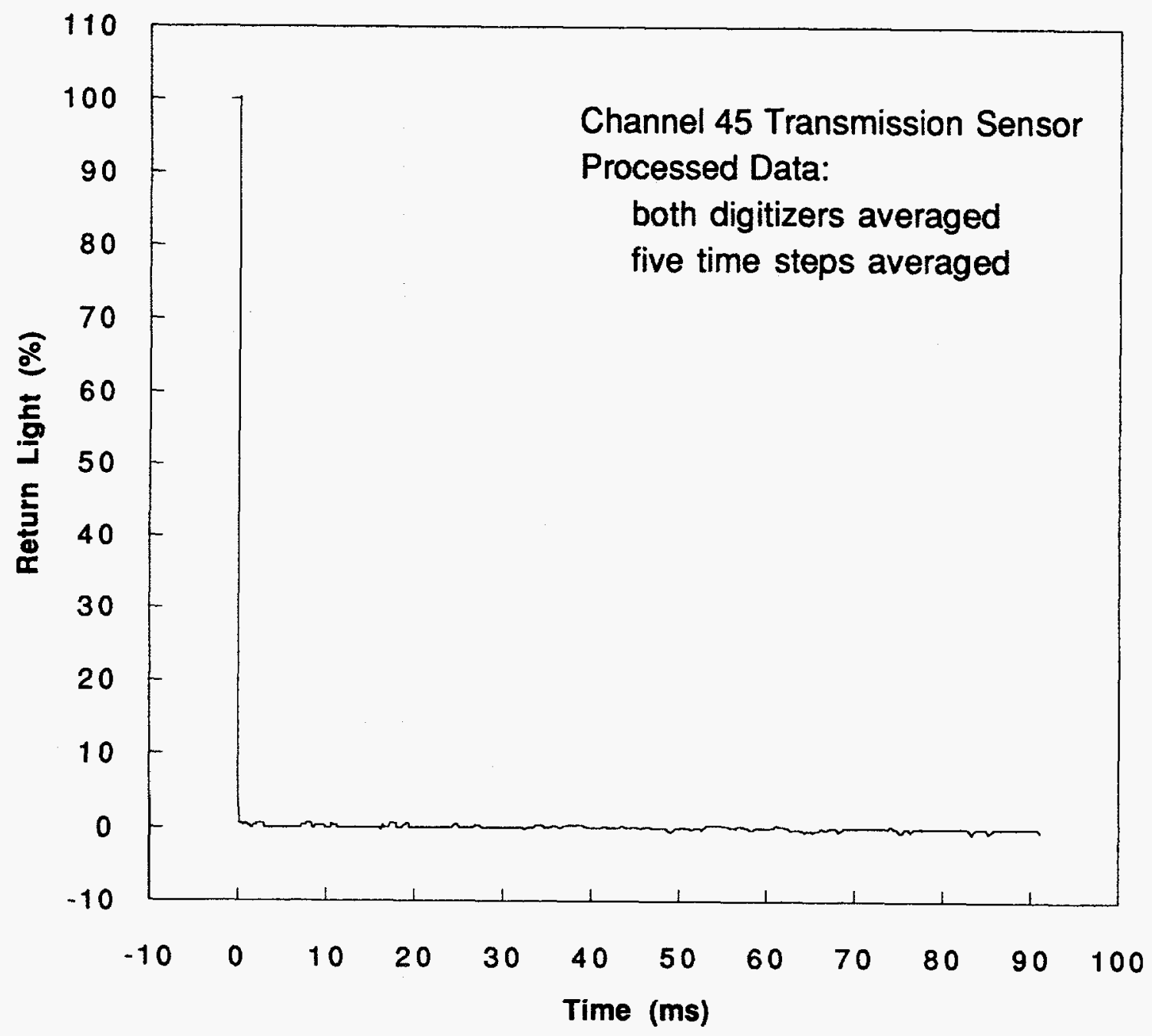

Figure 11. Data for the channel 45 two-fiber transmission gauge averaged over redundant digitizers and over five raw time steps. The $60 \mathrm{~Hz}$ noise has been subtracted. 


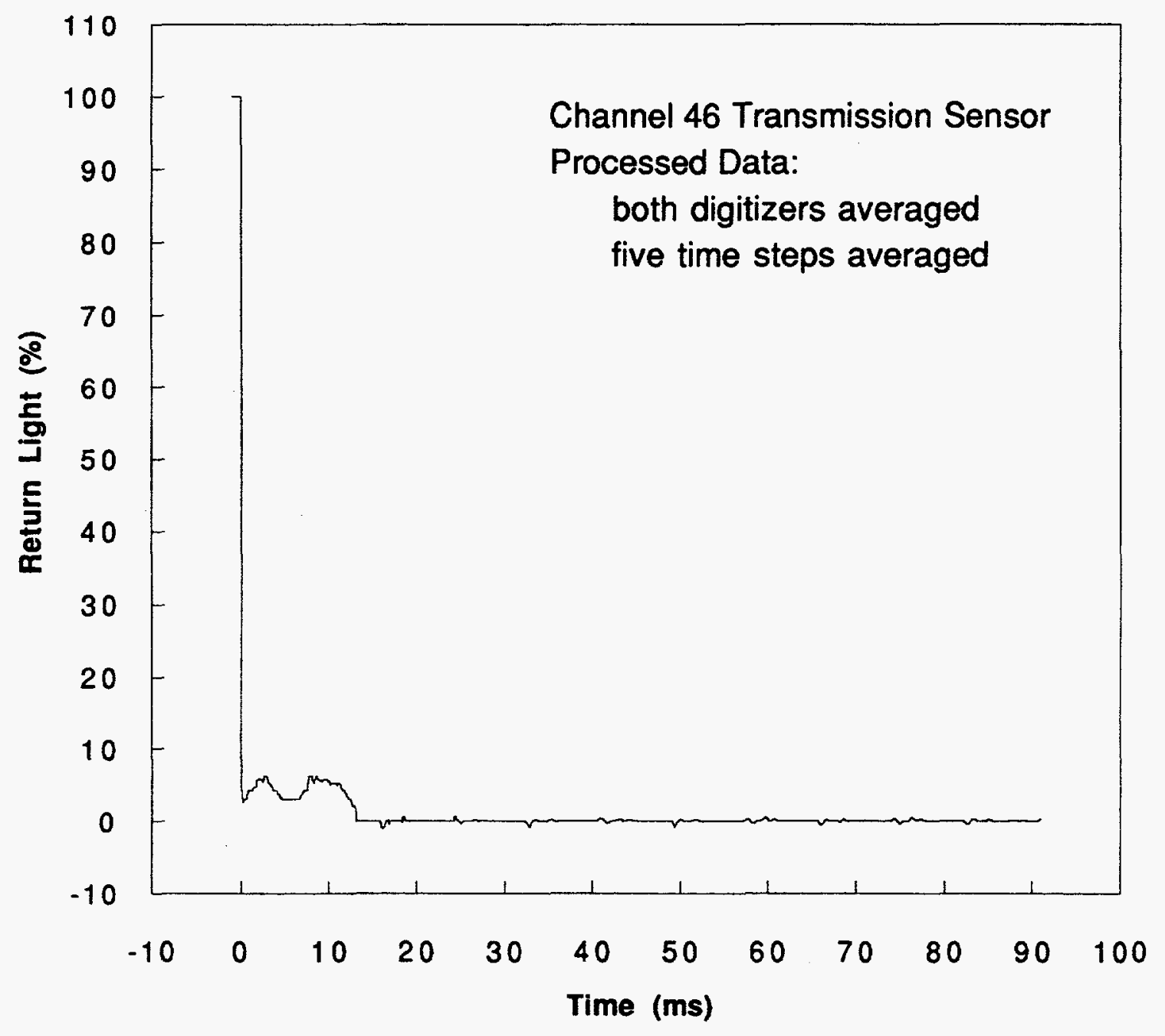

Figure 12. Data for the channel 46 two-fiber transmission gauge averaged over redundant digitizers and over five raw time steps. The $60 \mathrm{~Hz}$ noise has been subtracted. 


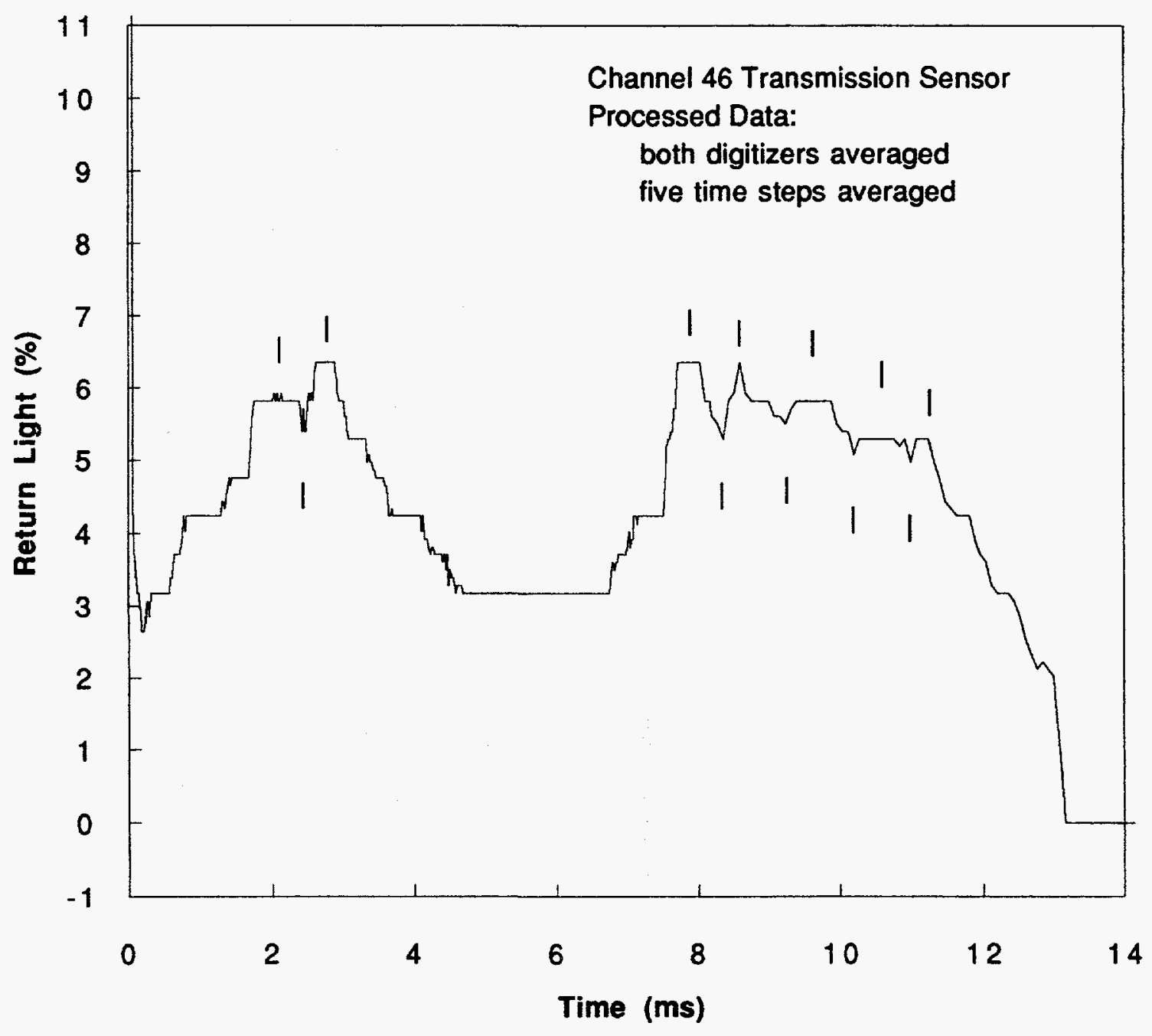

Figure 13. Early time data (noise subtracted; averaged over redundant digitizers and over five raw time steps) for the channel 46 two-fiber transmission gauge. Tick marks have been added to suggest possible locations of modulation maxima and minima. 
sensors in bench tests, is why the apparent modulation in the event data is so shallow. The stretch-wire data indicate that several grid holes necessarily passed by the optical system during the 1 to $4 \mathrm{~ms}$ time period so that the maxima and minima indicated by the tick marks added to Fig. 13 are the more appropriate modulations to consider than the overall double-humped structure. One encouraging conclusion independent of the mysteries is that light could indeed pass through the sensor which implies the darkening of the internal optical components over and above that of the supply and signal fibers is not a major addition to the overall problem of fiber darkening.

\section{Summary and Conclusions}

Plausible explanations of the optical displacement sensor data taken in tandem with that for the Hunters Trophy UGT pressure cell impulse gauge materials studies have been given in this report and offer hope for future developments. The fiber loop background data provide useful information on fiber performance, although a thorough analysis that attempts to explain absolute magnitudes would require knowledge of radiation fluxes at all relevant points in the experimental test chamber and in the line of sight drift which is beyond the scope of this report. The behavior seen is consistent with that of more controlled laboratory studies not hampered by the difficulties of underground nuclear tests and results from such studies can undoubtedly be applied directly to underground experimental designs.

The magnitude of the fiber darkening in the severe environment of the Hunters Trophy test chamber is a major but not insurmountable problem for operation of systems like our transmission style fiber-optic impulse gauges in such locations. After the initial fiber darkening of output signal levels in one of the optical sensor systems, the existence of later signals comparable in magnitude to the fiber background loop implies that darkening of the internal optical components was not a major additional problem. The systems we used as light sources had large reserve capacities which could respond rapidly to changes in their control voltages. Had there been a method in place to increase the laser diode output power for all transmission systems in response to the attenuation in the background fiber loop, we could have generated nearly the full response expected from an undarkened fiber system within a few milliseconds following the start of the event. Appendix $C$ provides a detailed example of how such a feedback-stabilized system could be applied in a future UGT event utilizing transmission-style displacement sensors.

For our reflective system gauges with their required offsets and high gains in the instrumentation, the fiber darkening was devastating and such systems should probably not be pursued for use in UGT environments. As a point of interest only, evidence of the expected fiber luminescence was apparently seen in the data from these systems before fiber darkening was fully developed.

\section{Acknowledgments}

The authors benefited from the support of many people during this study, among them the following. Erwin Schwegler, Jr. served as the Project Officer for the overall materials properties experiments fielded by Los Alamos National Laboratory on the Hunters Trophy event. Richard Scammon was instrumental in guiding the overall fielding effort for this experimental program and was an essential link in the data reduction process. Ken Spicochi provided valuable support to the design effort and to fielding the systems. Connon Odom oversaw the electronics and data recording support required at the Nevada Test Site. Richard Schiltz and Landon Hughes provided invaluable help with the fiber-optic components and links. The efforts of Michael $D$. Garcia in championing this work with the funding offices involved were critical to the success of this project and are greatly appreciated. This work was supported by the Department of Energy under contract W-7405-ENG-36. 


\section{Appendix A}

\section{Procedures Used to Subtract Periodic Noise from Raw Data}

In the raw calibrations, event data, and dry runs for the gauges using transmission style sensors, by far the largest noise component was a repetitive pattern which occurred at $60 \mathrm{~Hz}$. Its amplitude did not vary significantly for various constant input levels, so this noise was attributed to the output stages of the optical receiver systems. A third optical receiver system of the same type was used on the round trip fiber background system but had no detectable periodic noise and had only a constant zero-level correction applied to it. The four records of interest for event data (two systems, each redundantly recorded) were independently corrected for periodic noise before further processing using the procedures described in this appendix.

Perhaps fortuitously but none-the-less conveniently, the data for the gauges of interest indicated essentially zero light transmission at late times after the event trigger, from well before $40 \mathrm{~ms}$ after the trigger to the end of the records at slightly over $91 \mathrm{~ms}$. We thus chose to fit the data from $40 \mathrm{~ms}$ to the end of the records to the form

$$
Z(t)=A+B t+C \cos ^{n}\left[\omega_{60}(t-D)\right]
$$

where $\omega_{60}$ is the angular frequency for $60 \mathrm{~Hz}$ in units appropriate for the time units being used and A, B, C, n, and D are the parameters for the fits. The periodic noise alternated between positive and negative, so $n$ was assumed to be a positive odd integer. After several trial n's were tested using least-squares fits for the other parameters involved, $\mathbf{n}$ was fixed at seven for all cases. The other four parameters for all cases were then found by a detailed least-squares fit to all the data points in a record beyond $40 \mathrm{~ms}$. Results are tabulated in Table A1. The parameter A does not represent noise but rather the value of the deliberate offset introduced in the receiver system outputs. The parameter B was introduced to approximate the effects of long term drift in the system levels, known to be present but on slow time scales relative to the measurements of interest (the small values found for the B's confirmed this assumption).

The digitizers involved were all nominally set up the same and thus for a single output split to two independent units one expects similar results within small differences in the nominal conversion gains, zero levels, and relative phases of the timing pulses.

Table A1. Zero-level fits of $Z(t)=A+B t+C \cos ^{7}\left[\omega_{60}(t-D)\right]$ to event data for the Hunters Trophy optical impulse gauge development systems sensed with transmission optics.

\begin{tabular}{|c|c|c|c|c|c|c|}
\hline \multicolumn{2}{|c|}{ SYSTEM } & \multicolumn{5}{c|}{ FIT PARAMETERS } \\
\hline $\begin{array}{c}\text { electronics } \\
\text { channel } \\
\text { number }\end{array}$ & digitizer & $\begin{array}{c}\text { A } \\
\text { (counts) }\end{array}$ & $\begin{array}{c}\mathrm{B} \\
\text { (cnts/ms) }\end{array}$ & $\begin{array}{c}\mathrm{C} \\
\text { (counts) }\end{array}$ & $\begin{array}{c}\mathrm{D} \\
(\mathrm{ms})\end{array}$ & $\begin{array}{c}\text { standard } \\
\text { deviation } \\
\text { (counts) }\end{array}$ \\
\hline 45 & $1508 / 20$ & 9.4894 & 0.0162 & 1.350 & 0.907 & 0.3634 \\
\hline 45 & $1510 / 20$ & 9.8115 & 0.0129 & 1.355 & 0.878 & 0.3571 \\
\hline 46 & $1508 / 21$ & 34.0612 & 0.0004 & 2.005 & 0.921 & 0.2661 \\
\hline 46 & $1510 / 21$ & 33.9338 & 0.0006 & 2.094 & 0.934 & 0.2322 \\
\hline 47 & $1508 / 22$ & 9 & $-0-$ & $-0-$ & n/a & n/a \\
\hline 47 & $1510 / 22$ & 9 & $-0-$ & $-0-$ & n/a & n/a \\
\hline
\end{tabular}


The fit parameters of Table A1, extracted independently for each digitizer of both systems, indeed agree very well. Considering that the analog signals are rounded to integer counts in the digitizers, differences of up to 1 count in the $A$ values would be reasonable while in fact they are 0.32 and 0.13 counts respectively for the two cases. The slope parameters B for channel 46 are essentially zero while those for channel 45 correspond to a change of slightly over one count over the entire record length and agree with each other to within 0.3 counts over the record length. The amplitudes $C$ extracted for the $60 \mathrm{~Hz}$ noise agree very well between digitizers in each of the channels. The phase times D found for the $60 \mathrm{~Hz}$ noise agree to $29 \mu \mathrm{s}$ and $13 \mu \mathrm{s}$ respectively from fits in a region where the sampling rate for the raw digitizer records is $65 \mu$ s/point.

In order not to introduce a deceptive and artificial precision to the noise-corrected records, we integerized these records after applying the noise and zero-level correction from the fits by using

$$
\text { corrected counts at } t=\text { integer value }[0.5+(\text { raw counts at } t)-Z(t)]
$$

as our starting records for further analysis. These corrected initial data records have the same intrinsic graininess as the raw data but are much cleaner in appearance. From this point onward, averaging over digitizers or points in a record is considered an acceptable method of further smoothing the data to better see its features. 


\section{Appendix B}

\section{Computer Data Processing Details}

The raw data from these fiber-optic sensor experiments were initially recorded in the Defense Nuclear Agency (DNA) data acquisition system in Area 12 at the Nevada Test Site. Extraction of the relevant subsets of data from the overall DNA records for Hunters Trophy was handled [11] in conjunction with that for the overall Los Alamos experimental program. Data files were transferred to the Los Alamos Design Engineering (WX) Division cluster of Digital Corporation VAX computers and then subsequently to IBM compatible personal computer files. During these steps, the known time calibrations were applied to the data. However, we requested that the known voltage calibrations not be applied since they were not germane to the subsequent analysis and we preferred to begin final processing using the raw digitizer amplitude data, as discussed in the main text. The IBM compatible files were written out in ASCII form on 3.5 inch floppy disks and an Apple Corporation file transfer utility was used to read them into the Macintosh Ilcx personal computer used for final data reduction. At the time this report was prepared, these files were still being retained with the file names given in Table B1 in rows labeled "IBM-PC file."

After the IBM files were transferred into the Macintosh IIcx system, they were read with Microsoft Corporation's WORD 5.0 program and processed to remove superfluous characters and to reformat the material into a form, saved with the file names given in the rows labeled "WORD 5.0 file" in Table B1, that could be readily transferred into Microsoft EXCEL 4.0 file formats. Detailed instructions for this process are recorded in the project notebook [14]. Subsequent processing of the data using EXCEL 4.0 is described in the main text and in Appendix A. Within a minor exception to be discussed shortly, the EXCEL file names for the final data sets as used for figures in this report are given in Table B1. Any of these files can be copied to a 3.5 inch $2 \mathrm{HD}$ micro floppy disk, but only the smaller files involving little processing (dry runs and reflection sensor gauges) will fit on a 2DD disk. The channel 45 and 46 files in their full forms, currently with file names "DATAch45extnd" and "DATAch46extnd" on the hard disk versions, were too large to fit on $2 \mathrm{HD}$ disks and thus the final column used to average over digitizers and five time points (for main text figures 11 through 13) was dropped for the versions archived on floppy disks and named in Table B1. Once the data

Table B1. Guide to computer file names used in the final processing steps in a Macintosh IIcx personal computer. As of the publication date for this document, these files were being maintained by the authors and can be copied to $2 \mathrm{HD}$ micro floppy disks.

\begin{tabular}{|c|c|c|c|c|c|c|c|c|}
\hline $\begin{array}{l}\text { DNA DES-1 Digitizer, } \\
\text { unit/channel designation }\end{array}$ & $1508 / 20$ & $1510 / 20$ & $1508 / 21$ & $1510 / 21$ & $1508 / 22$ & $1510 / 22$ & $1508 / 23$ & $1510 / 23$ \\
\hline $\begin{array}{l}\text { Electronics support group } \\
\text { channel label }\end{array}$ & \multicolumn{2}{|l|}{45} & \multicolumn{2}{|c|}{46} & \multicolumn{2}{|l|}{47} & 48 & 57 \\
\hline \multicolumn{9}{|l|}{ EVENT DATA: } \\
\hline IBM-PC file & F81D.DAT & F11D.DAT & F82D.DAT & F12D.DAT & F83D.DAT & F13D.DAT & F84D.DAT & F14DDAT \\
\hline WORD 5.0 file & F81D.WRD & FIID.WRD & F82DWRD & F12D.WRD & F83D.WRD & F13D.WRD & F84D.WRD & F14D.WRD \\
\hline EXCEL 4.0 file & \multicolumn{2}{|c|}{ DATAch45 } & \multicolumn{2}{|c|}{ DATAch46 } & \multicolumn{2}{|c|}{ DATAch47 } & \multicolumn{2}{|c|}{ DATAch $48 \& 57$} \\
\hline \multicolumn{9}{|l|}{ CAIIBRATION: } \\
\hline IBM-PC file & F81C.DAT & FIIC.DAT & F82C.DAT & F12C.DAT & F83C.DAT & F13C.DAT & F84C.DAT & F14C.DAT \\
\hline WORD 5.0 file & F81C.WRD & & F82C.WRD & & F83C.WRD & & F84C.WRD & \\
\hline EXCEL 4.0 file & CAISdg1508 & & CALSdg1508 & & CALSdg1508 & & CALSdg1508 & \\
\hline \multicolumn{9}{|l|}{ 9/10/92DRY RUN: } \\
\hline IBM-PC file & A81D.DAT & & & & & & & \\
\hline WORD 5.0 file & A81D.WRD & & & & & & & \\
\hline EXCEL 4.0 file & DRYch45 & & & & & & & \\
\hline \multicolumn{9}{|l|}{ 9/14/92 DRY RUN: } \\
\hline IBM-PC file & & & & & B83D.DAT & & & \\
\hline WORD 5.0 file & & & & & B83D.WRD & & & \\
\hline EXCEL 4.0 file & & & & & DRYch47 & & & \\
\hline
\end{tabular}


is read back onto a hard disk, it is straightforward to restore the column by inserting the appropriate formula.

Table B2 shows selected portions of the EXCEL 4.0 spreadsheet data file "DATAch46extnd" and illustrates the type of processing done using these files as well as typical file structure. The first column, also repeated as the eighth for convenience in generating plots, contains the data point times as converted to milliseconds from the DNA records. Columns 2 and 3 contain the raw amplitude data from the two redundant digitizers. Note that the large initial signal drop occurs over five time points at $4 \mu \mathrm{s} / \mathrm{pt}$ without continuing effects thus confirming the assertions in the main text concerning transmission system time responses. Columns 5 and 6 , rows 3316 through 4102 , are the raw data of columns 2 and 3 minus the respective fits to these data described in Appendix A. Rows 3308 through 3311 of these columns contain the respective fit parameters, as labeled by the entries in column 7 . The mean values for the fits are given in row 3305 and, as should be, are zero to better than the least significant digit in the A parameters used to fit overall offset. The standard deviations of the fits are calculated in row 3306 for the two respective columns. These were minimized by manual searches through the parameter spaces for each column.

In columns 9 and 10, rows 7 through 4102, we calculate the "signal levels" SA and $\mathrm{SB}$ for each time point in the initial records by taking the corrected counts CCA and CCB calculated as described in Appendix A using the fit parameters from columns 5 and 6 respectively and applying a normalization factor $N$ input as the row 4 entry of the column (indicated by the label in column 8, row 4). We also allow an overall level shift $\mathrm{L}$ before normalization to be input into row 3 of the respective columns but have set it to zero for the calculated signals here. The normalizations for the table here are chosen to give signals which are nominally the light transmission as a percentage of that seen initially before the event.

Columns 12 through 15 contain contractions and averages over subsets of the times and signal levels to reduce the data sets to manageable sizes for plotting and for smoothing to enhance features otherwise obscured by the low resolution of the digitizers. Rows 5 and 6 of these columns are arbitrary extensions of the data added to improve the graphical clarity for some of the plots. Row 7 contains the first raw data time point (taken to be $t=0$ ) and its corresponding signal levels without other manipulation. Rows 8 through 826 contain parsed or averaged data. The row 8 , column 12 time point entry corresponds to the fourth raw data point and subsequent entries in this column are parsed to every fifth raw data point beyond the preceding entry. Columns 13 and 14 are simply the parsed signal levels in the two digitizers corresponding to the parsed time data points. The entries in column 15 from row 8 onward are calculated by averaging both digitizers together and averaging the data points from the full data sets for the time entry in column 12 with those for the two times on either side of it that have been parsed out of column 12. The EXCEL formulas needed to accomplish the above calculations are recorded in the project notebook [14].

The structure of the spreadsheet "DATAch45extnd" is identical to that just described. The EXCEL 4.0 spreadsheets for the other data sets are less complicated because they do not contain the noise fits and corrections. Their structures should be evident from the internal column headings provided with them. 
Table B2. Selected portions illustrating the overall layout of the channel 46 spreadsheet as used for final data reduction.

\begin{tabular}{|c|c|c|c|c|c|c|c|c|c|c|c|c|c|c|c|}
\hline & 7 & 2 & 3 & 4 & 5 & 6 & 7 & 8 & 9 & 10 & 11 & 12 & 13 & 14 & 15 \\
\hline 1 & thme & raw counts & raw counts & & dptz 1508 & dgtz 1510 & & tims & $S A=$ & $\mathrm{SB}=$ & & parsed & parbed & parsed & averape over \\
\hline 2 & (ms) & datz 1508 & dotz 1510 & & \{ch 21\}: & (ch 21) & & $(m s)$ & CCA+LYN & $(\mathrm{CCB}+\mathrm{L}) / \mathrm{N}$ & & $\operatorname{tin} \theta$ & SA & SB & SA\& SB \\
\hline 3 & & (ch 21) & (ch 21) & & fit to noise & fit to noise & & L- & 0 & 0 & & $(m s)$ & & & 85 time pts \\
\hline 4 & & $=A A$ & $=\overline{A B}$ & & beyond $40 \mathrm{~ms}$ & beyond $40 \mathrm{~ms}$ & & $N=$ & 0.95 & 0.94 & & & & & \\
\hline 5 & & & & & -800 below & -800 bolow & & & & & & -1 & 100 & 100 & 100 \\
\hline 6 & & & & & & & & & & & & -0.032 & 100 & 100 & 100 \\
\hline 7 & 0 & 130 & 129 & & & & & 0 & 100 & 100 & & 0 & 100 & 100 & 100 \\
\hline 8 & 0.004 & 130 & 129 & & & & & 0.004 & 100 & 100 & & $\overline{0.012}$ & 100 & 100 & 100 \\
\hline 9 & 0.008 & 130 & 129 & & & & & 0.000 & 100 & 100 & & 0.032 & 100 & 100 & 100 \\
\hline 10 & 0.012 & 130 & 129 & & & & & 0.012 & 100 & 100 & & 0.052 & 10.536 & 10.638 & 10.208287 \\
\hline $\overrightarrow{11}$ & 0.016 & 130 & 129 & & & & & 0.016 & 100 & 100 & & 0.072 & 4.2105 & 4.2553 & 4.2329227 \\
\hline 12 & 0.02 & 130 & 129 & & & & & 0.02 & 100 & 100 & & 0.002 & 4.2105 & 3.1915 & 3.7010078 \\
\hline 13 & 0.024 & 130 & 129 & & & & & 0.024 & 100 & 100 & & 0.112 & 3.1579 & 3.1915 & 3.3852164 \\
\hline 14 & 0.028 & 130 & 129 & & & & & 0.028 & 100 & 100 & & 0.132 & 3.1579 & 3.1915 & 3.174692 \\
\hline 15 & 0.032 & 130 & 129 & & & & & 0.032 & 100 & 100 & & 0.152 & 3.1579 & 3.1915 & 3.174692 \\
\hline 16 & 0.038 & 130 & 129 & & & & & 0.036 & 100 & 100 & & 0.172 & 3.1579 & 3.1915 & 2.8610261 \\
\hline 17 & 0.04 & 130 & 129 & & & & & 0.04 & 100 & 100 & & 0.192 & 3.1579 & 2.1277 & 2.6427772 \\
\hline 18 & 0.044 & 84 & 94 & & & & & 0.044 & 51.57895 & 62.76596 & & 0.212 & 3.1579 & 2.127 & 26427772 \\
\hline 10 & 0.048 & 47 & 49 & & & & & 0.048 & 12.63158 & 14.89362 & & 0.232 & 3.1579 & 2.1277 & 2.7491601 \\
\hline $\boldsymbol{\mathbf { m }}$ & 0.052 & 45 & 45 & & & & & 0.052 & 10.52632 & 10.6383 & & 0.252 & 3.1579 & 2.1277 & 2.9619261 \\
\hline 21 & 0.056 & 41 & 41 & & & & & 0.056 & 6.315789 & 6.382979 & & 0.272 & 3.1579 & 3.1915 & 3.0683091 \\
\hline 2 & 0.06 & 38 & 38 & & & & & 0.06 & 3.157895 & 3.191489 & & 0.292 & 3.1579 & 3.1915 & 2.8555431 \\
\hline 23 & 0.064 & 39 & 39 & & & & & 0.064 & 4.210526 & 4.255319 & & 0.312 & 3.1579 & 3.1915 & 3.174692 \\
\hline 2 & 0.068 & 39 & 39 & & & & & 0.068 & 4.210526 & 4.255319 & & 0.332 & 3.1579 & 3.1915 & 3.174692 \\
\hline 25 & 0.072 & 39 & 39 & & & & & 0.072 & 4.210526 & 4.255319 & & 0.352 & 3.1579 & 3.1915 & 3.174692 \\
\hline
\end{tabular}

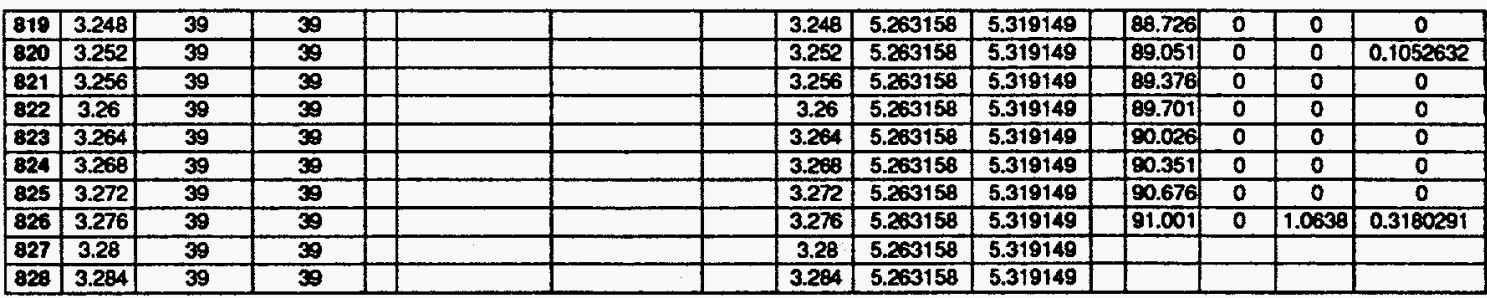

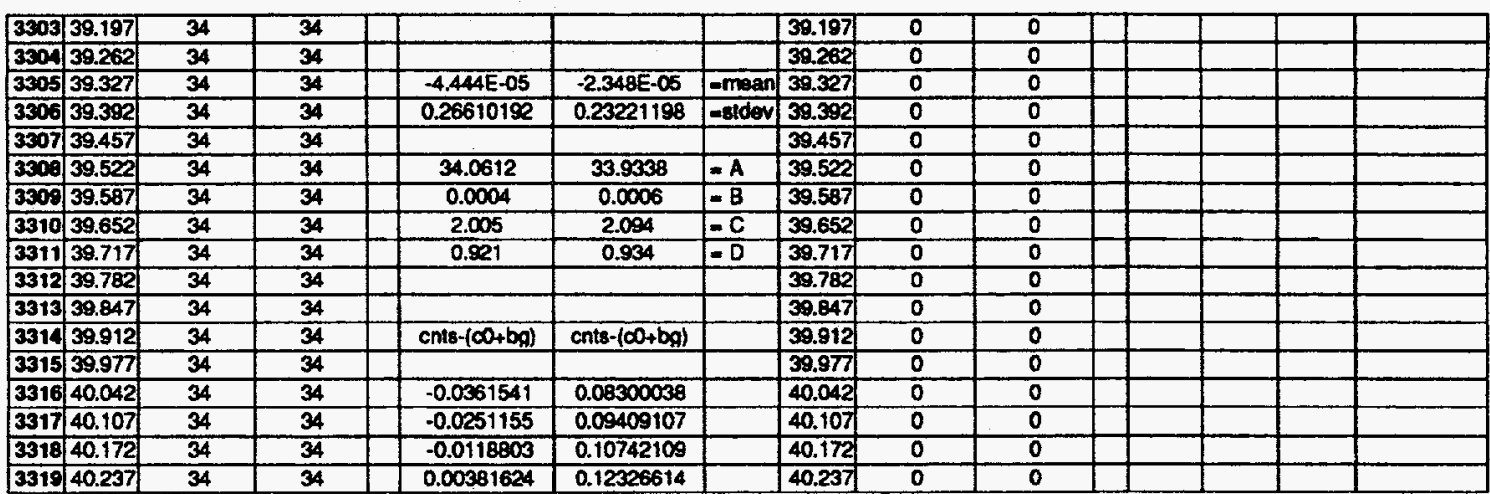

\begin{tabular}{|c|c|c|c|c|c|c|c|c|c|c|c|c|}
\hline 4004 & 90.611 & 34 & 34 & 0.13492558 & 0.2469212 & 90.611 & 0 & 0 & & & & \\
\hline 4005 & 90.676 & 34 & 34 & 0.17369699 & 0.28655304 & 90.676 & 0 & 0 & & & & \\
\hline 4006 & 90.741 & 34 & 34 & 0.21659823 & 0.33047613 & 90.741 & 0 & 0 & & & & \\
\hline 4097 & 90.806 & 34 & 34 & 0.26370652 & 0.37878097 & 90.806 & 0 & 0 & & & & \\
\hline 4000 & 90.871 & 34 & 34 & 0.31504746 & 0.43150555 & 90.871 & 0 & 0 & & & & \\
\hline 4000 & 90.936 & 34 & 34 & 0.37059034 & 0.48962994 & 90.936 & 0 & 0 & & & & \\
\hline 4100 & 81.001 & 34 & 34 & 0.43024383 & 0.55007172 & 91.001 & 0 & 1.06383 & & & & \\
\hline 4101 & 91.066 & 34 & 34 & 0.49385265 & 0.61568231 & 91.066 & 0 & 1.06383 & & & & \\
\hline 4102 & 91.131 & 34 & 33 & 0.56119532 & -0.31475566 & 91.131 & 1.052632 & 0 & & & & \\
\hline 4103 & & & & & & & & & & & & \\
\hline
\end{tabular}




\section{Appendix C}

\section{Sample Proposal for a Feedback Stabilized System}

Results from the tests of fiber-optic displacement sensors on Hunters Trophy clearly indicated that the simple systems used without extra radiation shielding around the fibers were not adequate to overcome fiber darkening well enough to make the measurements desired. However, the results obtained in the test give hope that future measurements in similar environments might be done with minor modifications to the transmission system electronics without requiring any extra efforts at shielding the fibers by the simple expedient of using a light path feedback loop to compensate for the fiber darkening. As indicated by the dry run data presented in the main text, response times of the optoelectronics are quick (tens of microseconds) compared to the mechanically modulated signals of interest in these displacement gauges. Since laser diode light sources with large excesses of light compared to that required to drive such systems exist (the Hunters Trophy transmission system light sources were run at only 3\% of capacity), existing optoelectronics could be used for the majority of the component parts of such systems. As an example of one such scheme, Fig. C1 shows a proposal for operating 12 digitally modulated mechanical displacement sensors using two FO-9 fiber cables (16 fibers total) of the type used on Hunters Trophy. The particular scheme shown assumes operation inside a vacuum vessel which is itself contained in a sealed experimental alcove.

In Fig. C1, two independent light sources are coupled after transmission to the sensor area in order to give redundancy in the light supply for all gauge systems. The intent would be to run the gauges and the two unmodulated optics background paths in as similar radiation environments as possible and to adjust the feedback circuitry to force the return light in the background paths to remain constant up to the limits of full laser diode output from both sources. Background paths would ideally have all the same optical components as the gauges although the Hunters Trophy data indicate the fiber alone is probably the dominant factor in signal loss. Except for the feedback circuitry, all the electronics required for such a system already exist in commercial form. In the simplest form, the feedback system could be an appropriately selected attenuator placed between the output of the optical receiver and the laser diode current control input built into some existing laser diode drivers. It is untested at present whether such a simple feedback method is stable when required to compensate for the large, sudden change of the magnitude seen in the fiber loop data in the main text. If this simple scheme is unstable, a modest development program ought to be able to produce some type of stable feedback system without difficulty. 


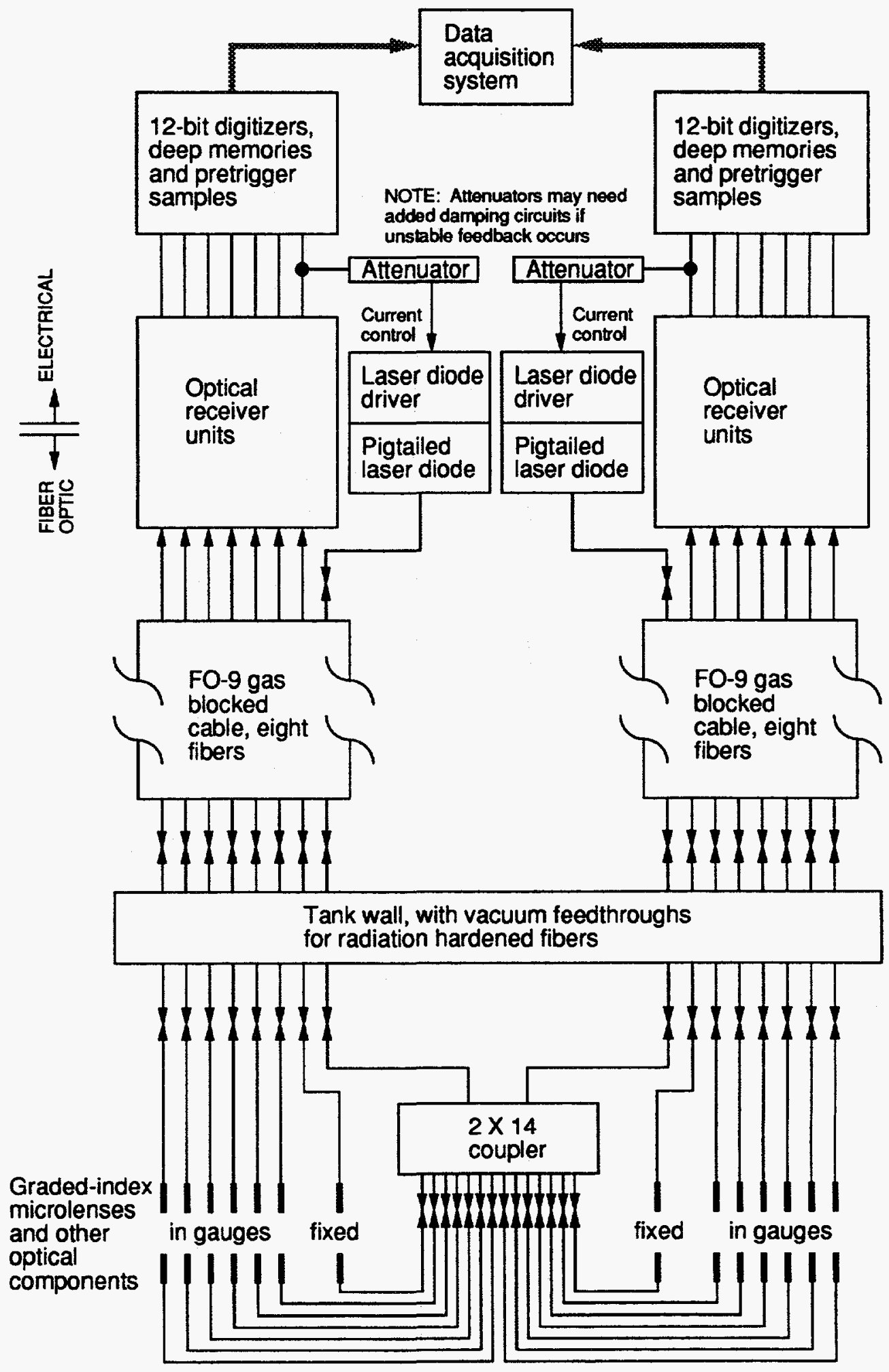

Figure $\mathrm{C} 1$. Electronics and optics for a feedback stabilized suite of twelve mechanically modulated displacement sensors driven by two redundant light sources. 


\section{References}

[1] R. S. Dingus et al. from Los Alamos National Laboratory, private communications. Mechanical details of the pressure cell gauges are given in the multiple sheet Los Alamos Scientific Laboratory drawings 18Y-134585 from 1977.

[2] Ray E. L. Green, Kenneth C. Spicochi, and Jack D. Thompson, "Fiber Optic Impulse Gauge Development," Los Alamos National Laboratory document LA-CP91-197 (1991, unclassified).

[3] Ray E. L. Green, Kenneth C. Spicochi, and Jack D. Thompson, "A Fiber Optic Sensor with a Five-Bit Digital Gray Code for Remote Measurement of Velocity/Displacement," in Proceedings of the Defense Nuclear Agency Instrumentation for Nuclear Weapons Effects Testing (INWET) Conference, 5-8 June 1989, DASIAC-89-77-V2, DNA procurement number DNA-001-88-C-0025 (ECI), pages 4-247 to 4-261 (1989).

[4] Ray E. L. Green, Kenneth C. Spicochi, Everett H. Horton, and Larry W. Berkbigler, "Ledoux Impulse Measurements and Target Surface Characterization Summary Report (U)," Los Alamos National Laboratory report LA-12280-MS (SFRD) (1992).

[5] Ray E. L. Green and James M. Bunch, "Ledoux Impulse and Pressure Data (U)," Eighth Biennial Nuclear Explosives Design Physics Conference (NEDPC) November 18-22, $1991(U)$, compiled by Raymond E. Hunter, Bruce T. Goodwin, M. Jolene Torres, and Patricia W. Mendius, Los Alamos National Laboratory document LA-12305-C, Vol. II (SRD), pages 311 to 321 (1992).

[6] R. E. L. Green, K. C. Spicochi, and E. H. Horton, section 6.3.2, pages 97 to 126, LEDOUX Preshot $(U)$, J. L. Richter, editor, Los Alamos National Laboratory document X-5-90-24 (SRD) (1990).

[7] Ray E. L. Green, Kenneth C. Spicochi, and Everett H. Horton, "Impulse Experiment Plans (U)," Los Alamos National Laboratory internal memorandum WX-11-90-112 (SFRD) (1990).

[8] Erwin Schwegler, Jr., Ray Green, and Richard Scammon, "Los Alamos Program Document for the Hunters Trophy Line-of-Sight Experiments (U)," Los Alamos National Laboratory document WX-11-91-42 (SRD) (1991).

[9] Connon Odom et al. from the Command, Control, and Communications Group of Los Alamos National Laboratory, private communications, 1992.

[10] Dick Schiltz, Landon Hughes, et al. from EG\&G/EM Las Vegas, private communications, 1992.

[11] Richard Scammon from the Analysis and Testing Group of Los Alamos National Laboratory, private communications, 1992 and 1993.

[12] T. A. Stringer and M. T. Lynch, "Radiation Effects Measurements on Optical Fibers in FBR and FXR Environments and Extrapolations to UGT Secondary Gamma and Neutron Environments," in Proceedings of DoD Fiber Optics '92, The 3rd Biennial Department of Defense Fiber Optics Conference, pages 39-45 (1992).

[13] T. Stringer, M. Lynch, and G. Paderewski, "Measurements of Optical Fiber Darkening and Luminescence in a Fast Burst Reactor Environment and Implications for UGT/LOS Applications (U)," Kaman Sciences Corporation document K-92408(R) (SRD) (1992).

[14] Ray E. L. Green, "Hunters Trophy: Fiber Optic Displacement Gauges," Los Alamos National Laboratory notebook number R007899. 